\title{
The role of phase shifts of sensory inputs in walking revealed by means of phase reduction
}

\author{
Azamat Yeldesbay ${ }^{1,2}$, Tibor Tóth ${ }^{1}$, and Silvia Daun ${ }^{1,2}$ \\ ${ }^{1}$ Heisenberg Research Group of Computational Neuroscience - Modeling Neural Network Function, Department of \\ Animal Physiology, Institute of Zoology, University of Cologne, Zülpicher Str. 47b, 50674 Cologne, Germany \\ ${ }^{2}$ Cognitive Neuroscience, Institute of Neuroscience and Medicine (INM-3), Research Centre Jülich, 52425 Jülich, \\ Germany.
}

Published in

Journal of Computational Neuroscience in June 2018, Volume 44, Issue 3, pp 313-339, DOI: https://doi.org/10.1007/s10827-018-0681-0

\begin{abstract}
Detailed neural network models of animal locomotion are important means to understand the underlying mechanisms that control the coordinated movement of individual limbs. Daun-Gruhn and Tóth, Journal of Computational Neuroscience 31(1):43-60 (2011) constructed an inter-segmental network model of stick insect locomotion consisting of three interconnected central pattern generators (CPGs) that are associated with the protraction-retraction movements of the front, middle and hind leg. This model could reproduce the basic locomotion coordination patterns, such as tri- and tetrapod, and the transitions between them.

However, the analysis of such a system is a formidable task because of its large number of variables and parameters. In this study, we employed phase reduction and averaging theory to this large network model in order to reduce it to a system of coupled phase oscillators. This enabled us to analyze the complex behavior of the system in a reduced parameter space.

In this paper, we show that the reduced model reproduces the results of the original model. By analyzing the interaction of just two coupled phase oscillators, we found that the neighboring CPGs could operate within distinct regimes, depending on the phase shift between the sensory inputs from the extremities and the phases of the individual CPGs. We demonstrate that this dependence is essential to produce different coordination patterns and the transition between them. Additionally, applying averaging theory to the system of all three phase oscillators, we calculate the stable fixed points - they correspond to stable tripod or tetrapod coordination patterns and identify two ways of transition between them.

Keywords: Central pattern generators; Inter-segmental coordination; Phase oscillator model; Stepping patterns; Transition; Speed control; 6-legged locomotion
\end{abstract}

\section{Introduction}

Insect walking has been the subject of many systematic investigations. To generate stable movements at different walking speeds and in various environments, a high degree of spatial and temporal coordination between the legs and body segments of an animal is needed.

One way to study the underlying mechanisms of coordinated walking is to build an appropriate model of the walking system based on relevant experimental data. In this approach, it is widely accepted that on the neural level, there are groups of interacting neurons, called central pattern generators (CPGs) that produce rhythmic activity (Delcomyn, 1980; Roberts and Roberts, 1983; Büschges, 2005; Katz, 2016). This oscillatory activity ultimately drives the muscles generating thereby rhythmic motor behavior. The coordinated 
movement is then achieved by mutual interaction of the CPGs, which also depends on the sensory feedback from the legs (for a review on the stick insect see Büschges and Gruhn (2007)).

An advantage of a detailed neural network model is that it can simulate the behavior of the locomotor system in various conditions and allows the analysis of the contribution of different functional elements of the model to producing a given behavior. However, because of the large number of variables and parameters of the system, it is a difficult task to investigate the model behavior by making use of analytical methods, only.

One of the methods to simplify and analyze a network of oscillatory systems is phase reduction theory (Guckenheimer and Holmes, 1983; Hoppensteadt and Izhikevich, 1997; Kuramoto, 1984). Using this method, a network of oscillators, where every oscillator is a high-dimensional system, can be reduced to a network of weakly coupled phase oscillators, where the state of every oscillator is described by just one phase variable. This reduction is achieved by approximation of the phase of each oscillator in a small neighborhood of its limit cycle. The result of the phase reduction is a model consisting of weakly coupled phase oscillators.

Due to their simplicity, coupled phase oscillators have been the first approach in many studies of animal locomotion, such as the model of swimmeret system of the crayfish (Skinner et al., 1997), swimming of lamprey (Cohen et al., 1982) and the model of coupled nonlinear oscillators of a hexapedal walking system (Collins and Stewart, 1993). Furthermore, the phase reduction theory together with the averaging theorem for weakly coupled oscillators (Guckenheimer and Holmes, 1983) was successfully used to reduce and analyze models with large number of variables, such as the model of cockroach locomotion (Proctor and Holmes, 2010; Proctor et al., 2010; Aminzare et al., 2018), the model of lamprey swimming (Massarelli et al., 2016), and the swimmeret system of the crayfish (Jones et al., 2003; Zhang and Lewis, 2016). Moreover, the approach of using coupled phase oscillators works well for systems with definite phase relations.

During locomotion, insects make use of a variety of walking patterns. Some of these patterns possess some form of symmetry. For instance, coordination patterns with four legs simultaneously on the ground are called tetrapod, whereas walking patterns with three legs on the ground at the same time tripod coordination patterns (Graham, 1985). It is known that slowly walking insects prefer the tetrapod coordination pattern to the tripod one, while fast walking insects have the opposite preference (Graham, 1985, 1972). Transition between these coordination patterns happens at a large enough change in the walking speed (Grabowska et al., 2012; Mendes et al., 2013).

To understand how coordination patterns are generated and stabilized, several models based on different approaches were developed. In one group of studies, a model of six-legged locomotion was constructed using certain rules of interaction between legs, obtained from behavioral experiments (Cruse, 1990; Dürr et al., 2004; Schilling et al., 2013). In other studies, neuro-mechanical models were constructed using experimental findings, such as a dynamic 3D-biomechanical model of the stick insect (Ekeberg et al., 2004; Von Twickel et al., 2012), or a neuro-mechanical model of cockroach locomotion (Holmes et al., 2006; Kukillaya and Holmes, 2007, 2009). As mentioned above, the latter model was reduced to one of coupled phase oscillators (Proctor and Holmes, 2010; Proctor et al., 2010).

In a previous work (Daun-Gruhn and Tóth, 2011) an inter-segmental network model was constructed that consisted of three cyclically interconnected CPGs. The CPGs generated the protraction-retraction rhythms of the horizontal leg movements during walking. Experimental data were used from the stick insect for the construction of the model. This model is an extension of that of Daun-Gruhn (2011). It is also the forerunner of other, more detailed models (Tóth et al., 2013a,b; Grabowska et al., 2015; Tóth et al., 2015; Tóth and Daun-Gruhn, 2016). More precisely, the model of Daun-Gruhn and Tóth (2011) comprises the CPGs of the protractor-retractor motor systems of the ipsilateral front, middle, and hind leg. The CPGs are connected via excitatory and inhibitory pathways, which are modulated by sensory feedback. The model can mimic the tripod and tetrapod coordination patterns, and the transition between them. Moreover, in this model two mechanisms controlling the transition were assumed: (i) via central drive, which controls the oscillatory period of the CPGs, and (ii) via a phase-dependent control mechanism, that changes the phase differences between the peripheral sensory signals and the oscillation of the segmental CPGs.

In the work presented here, we aimed at understanding the role of inter-segmental connections in, and the mechanisms underlying, the generation of the different coordination patterns and the transition between 
them during six-legged walking. To this end, we transformed the equations of the original inter-segmental network model to an equation system of phase oscillators by using the phase reduction theory and the averaging theorem. As a result, the 12 ordinary differential equations (ODEs) of the original model (DaunGruhn and Tóth, 2011) could be reduced to a system of 3 or 2 ODEs. The ODEs of phase variables could still capture the key oscillatory properties of the original model. This helped us a great deal to analyze the model of Daun-Gruhn and Tóth (2011) and to understand the role of the parameters of the system in generation of the various coordination patterns. Moreover, using the results of this study, we intend to perform a comparative investigation of locomotion models developed for different species of insects in the future in order to extract common features of motor control in them.

The work is structured as follows. In the first section (Section 2), we identify the parts of the intersegmental network model of Daun-Gruhn and Tóth (2011) that are essential for further analysis by means of the phase reduction method. In the Section 3, we introduce the phase reduction and the averaging theory in their general form and apply them to an isolated CPG and to a CPG with external synaptic connections. Then we derive the complete reduced system of coupled phase oscillators for the original model. The methods of analysis and the specificity of our approach will be presented in Section 3.5. In Section 4, we start with the analysis of a simpler model consisting of two coupled segmental CPGs in order to understand the properties of the inter-segmental connection with respect to the hypothetical control variables (peripheral sensory signals) of the model of Daun-Gruhn and Tóth (2011). Further, we demonstrate that the reduced system of three coupled phase oscillators can reproduce the results of the original model, such as stable tri- and tetrapod coordination patterns. Finally, we explain there the underlying mechanism of the transition between coordination patterns suggested in Daun-Gruhn and Tóth (2011): via the change in the phase-dependent control mechanism and by changing the oscillatory period of the CPGs.

\section{Inter-segmental network model of insect locomotion}

\subsection{General scheme}

The inter-segmental network model of Daun-Gruhn and Tóth (2011) is shown, in simplified form, in Fig. 1. The network consists of three segments that correspond to the ipsilateral front, the middle and the hind leg, respectively. Since Borgmann et al. (2007, 2009) found a weak neural connection between the contralateral legs, it seems justified to neglect them in the model. Thus it includes the connection between ipsilateral legs only. Influence from the contralateral side is neglected.

Each segment has a central pattern generator $(\mathrm{CPG})$ that is associated with the protractor-retractor muscles of the corresponding leg. The inter-segmental connections of the CPGs are modulated by peripheral sensory signals at the sensory interneurons (SINFL, SINML, SINHL). The sensory signals are denoted in Fig. 1 by $y_{F L}, y_{M L}$, and $y_{H L}$. The notations FL, ML, HL stand for front, middle and hind leg, respectively. The sensory signals originate in the levator-depressor neuro-muscular system of the corresponding leg and integrate several modalities of sensory information, i.e. position of the leg, ground contact or lift-off, and loading or unloading of the leg. The synaptic connections on the sensory interneurons are effective only, if sensory signals are also present at the same time.

The network, including the inter-segmental connections from pro- to meso-thoracic and from meso- to meta-thoracic segmental CPGs has been constructed using the results of the experimental works in the stick insect and locust (see the references in Daun-Gruhn and Tóth (2011)). The peripheral hypothetical connection from meta- to pro-thoracic ganglion which provided stability of the oscillation of the system was introduced by Daun-Gruhn and Tóth (2011).

\subsection{The model of a single CPG}

In the model, each CPG consists of two interneurons connected by inhibitory synapses. This type of model is called half-center oscillator (Brown, 1914; Wang and Rinzel, 1992; Calabrese et al., 2003; Daun et al., 2009). 


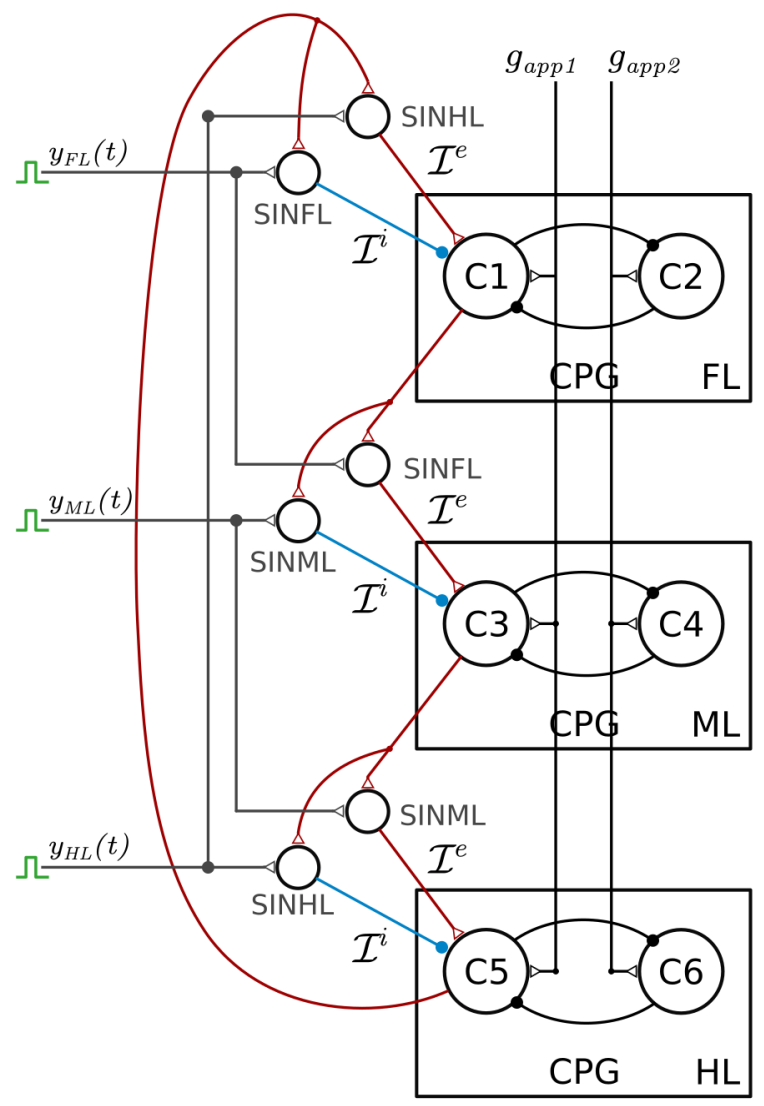

Figure 1: Scheme of inter-segmental network model adapted from Daun-Gruhn and Tóth (2011). Only parts essential for this paper are shown. Every CPG consists of two non-spiking interneurons, one of them is associated with the protractor, the other with the retractor muscle. The retractor CPG neurons C1, C3, and C5 are inter-segmentally and uni-directionally connected, the connections passing through the sensory interneurons SINFL, SINML, and SINHL. Moreover, the inter-segmental connections are cyclic. Sensory input signals $\left(y_{F L}(t), y_{M L}(t)\right.$, and $\left.y_{H L}(t)\right)$ originate from the levator-depressor neuro-muscular system of the corresponding leg and integrate several modalities of sensory information. The sensory synaptic input to a CPG neuron from the sensory interneuron of the leg in the same segment is inhibitory $\left(\mathcal{I}^{i}\right.$, blue lines), whereas that from the sensory interneuron of the leg in another, cyclically preceding, segment is excitatory $\left(\mathcal{I}^{e}\right.$, red lines).

The CPG model consists of two non-spiking Hodgkin-Huxley-type neurons connected by mutually inhibitory synapses (see Fig. 2 (a)). The full set of equations of the CPG is:

$$
\left\{\begin{aligned}
C_{m} \frac{d V_{1}}{d t} & =-\left(I_{N a P 1}+I_{L 1}+I_{s y n 1}+I_{a p p 1}\right)-\sum_{j} \mathcal{I}_{j}, \\
\frac{d h_{1}}{d t} & =\left(h_{\infty}\left(V_{1}\right)-h_{1}\right) / \tau\left(V_{1}\right), \\
C_{m} \frac{d V_{2}}{d t} & =-\left(I_{N a P 2}+I_{L 2}+I_{s y n 2}+I_{a p p 2}\right), \\
\frac{d h_{2}}{d t} & =\left(h_{\infty}\left(V_{2}\right)-h_{2}\right) / \tau\left(V_{2}\right),
\end{aligned}\right.
$$


where $I_{N a P 1}$ and $I_{N a P 2}$ are slowly inactivating $\mathrm{Na}^{+}$currents

$$
\begin{aligned}
& I_{N a P 1}=g_{N a P} m_{\infty}\left(V_{1}\right) h_{1} \cdot\left(V_{1}-E_{N a}\right), \\
& I_{N a P 2}=g_{N a P} m_{\infty}\left(V_{2}\right) h_{2} \cdot\left(V_{2}-E_{N a}\right),
\end{aligned}
$$

$I_{L 1}$ and $I_{L 2}$ are leak currents

$$
I_{L 1}=g_{L}\left(V_{1}-E_{L}\right), I_{L 2}=g_{L}\left(V_{2}-E_{L}\right),
$$

$I_{a p p 1}$ and $I_{a p p 2}$ are the central driving currents

$$
I_{a p p 1}=g_{a p p 1}\left(V_{1}-E_{a p p}\right), I_{a p p 2}=g_{a p p 2}\left(V_{2}-E_{a p p}\right) .
$$

Finally, the synaptic currents $I_{s y n 1}$ and $I_{s y n 2}$ between the CPG neurons are given as

$$
\begin{aligned}
& I_{\text {syn } 1}=g_{\text {syn }} s_{\infty}\left(V_{2}\right)\left(V_{1}-E_{\text {syn }}\right), \\
& I_{\text {syn } 2}=g_{\text {syn }} s_{\infty}\left(V_{1}\right)\left(V_{2}-E_{\text {syn }}\right) .
\end{aligned}
$$

The mutual synaptic connections between the CPG neurons are inhibitory. The inter-segmental synaptic currents $\mathcal{I}_{j}$ and the details of the synaptic connections between CPGs will be discussed below.

All steady state values of the activation variables $\left(m_{\infty}, h_{\infty}, s_{\infty}\right)$ are described by the following function:

$$
z_{\infty}(V)=\frac{1}{1+\exp \left(\gamma_{z}\left(V-V_{h z}\right)\right)}
$$

where $z$ is $m, n$, or $s$.

The time "constant" $\tau$, as a function of $V$, reads

$$
\tau(V)=\frac{1}{\varepsilon} \frac{1}{\cosh \left(\gamma_{\tau}\left(V-V_{h \tau}\right)\right)}
$$

where $\varepsilon$ is small.

The actual synaptic activation $s_{\infty}$ depends on the potential of the presynaptic cell, as shown in Eq. (2). In the model, all synaptic currents that arrive at a neuron are summed. The parameter $\gamma_{s}$ is chosen to be large. Thus, the CPG becomes a relaxation oscillator with the inhibitory synaptic connections modeled as "fast threshold modulation" (FTM) (Somers and Kopell, 1993, 1995). More specifically, it is an escape-type half-center oscillator. Nullclines and limit cycle of the CPG are shown in Fig. 2 (b). When one cell is active (depolarized, e.g. C1) the other cell is deactivated (hyperpolarized, e.g. C2). The V-nullclines of the cells which depend on the presynaptic potentials conveyed by the synaptic currents (Eq. (2)) are different (solid and dashed red lines). When the deactivated cell C2 approaches its left knee (solid red line) it becomes depolarized (activated). Thus it inhibits the cell C1, which becomes hyperpolarized (deactivated).

The parameters $g_{N a P}$ and $g_{L}$ are constant, whereas $g_{s y n}, g_{a p p 1}$, and $g_{a p p 2}$ can vary (see Appendix A). The conductances of the central drive, $g_{a p p 1}$, and $g_{a p p 2}$, define the period and duty factor of the CPG. The duty factor has originally been defined for a stepping leg as the ratio of the stance phase of the step to the whole stepping period:

$$
r_{0}=\frac{T_{\text {stance }}}{T_{\text {stance }}+T_{\text {swing }}}=\frac{T_{\text {stance }}}{T} .
$$

However, we shall also use this definition to characterize the periodic activity of the CPG neurons. Thus, stance phase in this context will mean the period when the CPG neuron associated with the retractor muscle, in short the 'retractor CPG neuron', is active (depolarized). The swing phase will analogously be defined for the 'protractor CPG neuron'.

Experimental observations show that for a certain coordination pattern of stepping there is a preferable ratio of the stance to the swing phase, i.e. a preferable duty factor. For example, the preferable ratio for tetrapod is about $3: 1$ and $r_{0} \approx 3 / 4$, whereas for tripod it is about 2:1 and $r_{0} \approx 2 / 3$, but always $r_{0}>0.5$ 
(Graham, 1985; Grabowska et al., 2012; Mendes et al., 2013). In Fig. 2 (c) and (d), two examples of the CPG activity with different conductance values of $g_{a p p 1}$, and $g_{a p p 2}$ are shown $\left(g_{a p p 1}=0.2350 \mathrm{nS}, g_{a p p 2}=0.1900 \mathrm{nS}\right.$ for tripod, and $g_{a p p 1}=0.2500 \mathrm{nS}, g_{a p p 2}=0.1855 \mathrm{nS}$ for tetrapod). They were chosen such that the CPG produces a stable oscillation with the preferable duty factors, just mentioned. We linearly changed $g_{a p p 1}$ and $g_{a p p 2}$ within these limits as shown in Fig. 3. If we decrease $g_{a p p 1}$ and simultaneously increase $g_{a p p 2}$ along the straight line as it is shown in Fig. 3 (a) with the gray arrow, then the period of the CPG oscillation decreases (Fig. 3 (b)) together with the duration of the stance phase (Fig. 3 (d)), whereas the duration of the swing phase remains almost constant. Thus the duty factor $r_{0}$ defined as the ratio of the stance phase to the period also decreases (Fig. 3 (c)).

\subsection{Coupling of the segmental CPGs}

The system of equations for a single CPG with external inter-segmental synaptic inputs to the retractor neuron is given in Eq. (1), where the index $j$ runs through all inter-segmental synaptic inputs. The whole network has three segments, and every segment is described by a system of equations of the form of Eq. (1).

In the inter-segmental network model (Fig. 1) every CPG is connected to another CPG by two synaptic pathways, one excitatory and one inhibitory, that pass through sensory interneurons (denoted as SINFL, SINML, and SINHL) modulated by sensory signals (denoted as $y_{F L}(t), y_{M L}(t)$, and $y_{H L}(t)$ ). Moreover, the inhibitory pathway is modulated by a peripheral sensory signal originating from the same segment, whereas the excitatory pathway is modulated by a peripheral sensory signal coming from the (cyclically) preceding segment. Note, that these pathways connect the retractor CPG neurons C1, C3, and C5, only (see Fig. 1). A model for sensory interneurons is not specified, but the assumption that the transmission of the intersegmental signal could only take place if it arrived at the same time as the corresponding sensory signal is made.

As mentioned above, the sensory signals encode peripheral sensory information. The sensory signal is non zero, if the leg has ground contact (is loaded), and zero, if the leg has no ground contact (is unloaded) (see Fig. 4). Thus in the inter-segmental network model, the sensory signals were modeled as rectangular functions of time with the duty factor $r_{y}=0.6$ (see Eq.(9) in Daun-Gruhn and Tóth (2011)):

$$
y(t)=\frac{a}{2}(1+\operatorname{sgn}(\sin (2 \pi(t / T-\phi)+\alpha)-\sin (\alpha))),
$$

where $\alpha=\pi\left(1 / 2-r_{y}\right)$, sgn is the sign function, and $a$ is the amplitude of the sensory signal, which can differ from segment to segment and from synapse to synapse. The active phase and the duty factor $r_{y}$ of the sensory signals were defined from the comparison of phase relations of the muscle activities at the different joints of a single leg (see Fig. 4 in Daun-Gruhn (2011), and Fig. 7 in Daun-Gruhn and Tóth (2011)). The period of the sensory signals $T$ is adjusted to the period of the CPG. Finally, the phase difference between the modulatory (sensory) signal and the activity of the segmental CPG at the modulatory synapses (enclosed in SINFL, SINML, and SINHL) are introduced as the phase shift $\phi$ (Eq. (6)). This phase shift also takes into account the phase relation between the levator-depressor activity and the protractor-retractor activity (Daun-Gruhn, 2011; Büschges, 2005), and the inter-segmental conduction delay. Moreover, Daun-Gruhn and Tóth (2011) hypothesized that the change of these phase shifts are the core of the mechanism of switching between coordination patterns. The animal initiates this switch via a phase-dependent control mechanism.

If CPG $k$ receives input from CPG $l(k, l=1,2,3 ; k \neq l$ stand for the thoracic segments where the CPGs reside) via the sensory interneurons, then the input currents are defined as

$$
\begin{aligned}
& \mathcal{I}_{k}^{e}=g_{\text {syn,k}}^{e} s_{\infty}\left(V_{\text {ret }, l}\right) a_{k}^{e} y_{l}^{e}(t)\left(V_{k}-E^{e}\right), \\
& \mathcal{I}_{k}^{i}=g_{\text {syn }, k}^{i} s_{\infty}\left(V_{\text {ret }, l}\right) a_{k}^{i} y_{k}^{i}(t)\left(V_{k}-E^{i}\right) .
\end{aligned}
$$

Here we have introduced new notations, substituting 1 for FL, 2 for ML, and 3 for HL. The upper indexes $i$ and $e$ denote inhibitory and excitatory synaptic connections, respectively. For example, $y_{k}^{e}(t)$ denotes the sensory signal modulating the excitatory pathway with amplitude 1 and originating from segment $k$ where $k=1,2$, or 3 (see Fig. 1). The reversal potentials $E^{e}=0.0 \mathrm{mV}$ and $E^{i}=-80.0 \mathrm{mV}$ are the same for 

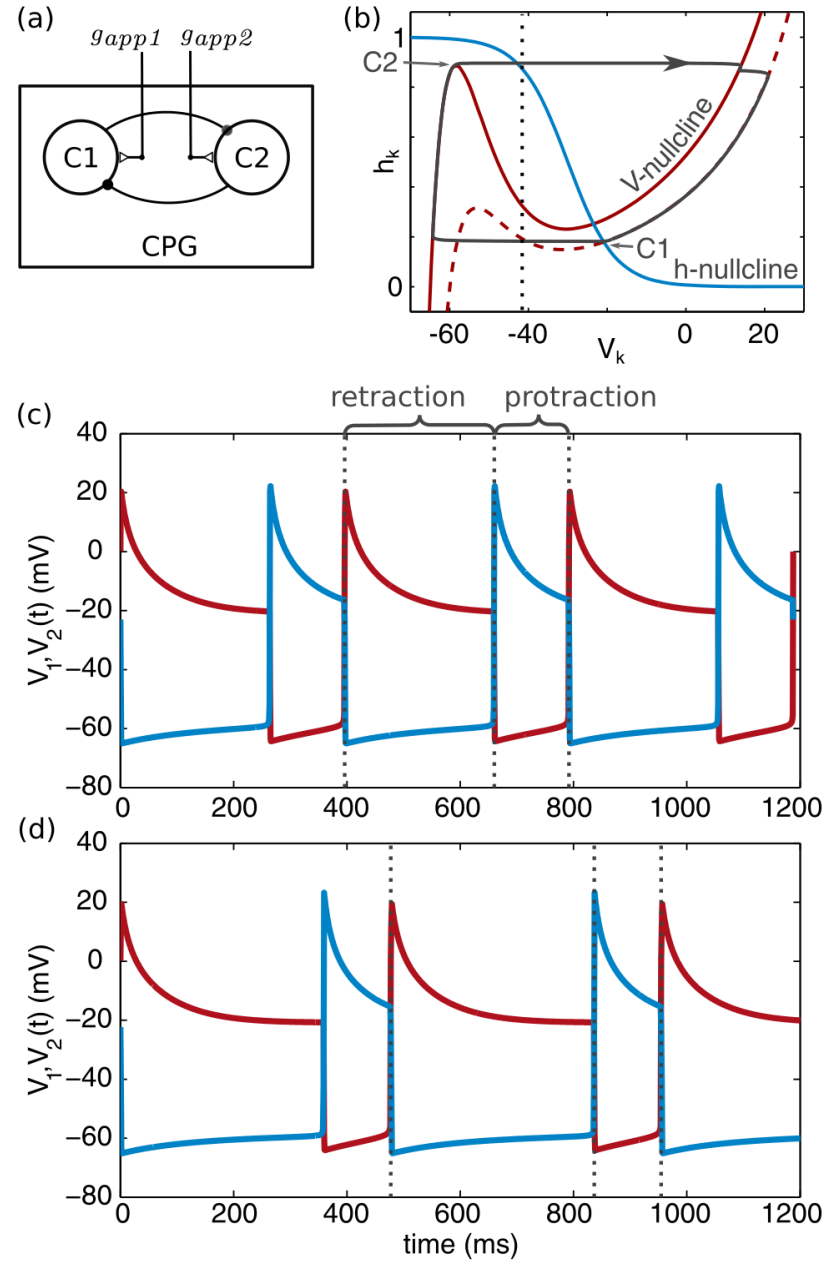

Figure 2: An isolated CPG. (a) Scheme of a CPG that consists of two non-spiking Hodgkin-Huxley-type neurons (C1 and C2) connected by mutually inhibitory synapses. $g_{a p p 1}$ and $g_{a p p 2}$ are conductances of the central drive. Period and duty factor of the CPG activity can be adjusted by changing the values of these parameters. (b) Nullclines and the limit cycle of a CPG in the symmetric case $\left(g_{a p p 1}=g_{a p p 2}\right)$. The solid blue line is the h-nullcline. The solid and dashed red lines are the $\mathrm{V}$-nullclines of the cells with and without inhibition, respectively. The solid black line is the limit cycle. Vertical dotted line is the threshold value $V_{h s}=-43.0 \mathrm{mV}$. C1 and C2 indicate the state of the cells just before switching between active and deactivated state. In the panels (c) and (d), examples of the time courses of the membrane potential of the retractor CPG neuron $\mathrm{C} 1$ (red) and the protractor CPG neuron C2 (blue) are shown for different parameter values: (c) $g_{a p p 1}=0.2350 \mathrm{nS}, g_{a p p 2}=0.1900 \mathrm{nS}$, the duty factor of the oscillation is $r_{0} \approx 2 / 3 ;(\mathrm{d})$ $g_{a p p 1}=0.2500 \mathrm{nS}, g_{a p p 2}=0.1855 \mathrm{nS}$, the duty factor is $r_{0} \approx 3 / 4$. The vertical dotted lines denote the boundaries between the stance phase and the swing phase.

all excitatory and inhibitory synapses. Finally, $g_{s y n, k}^{i}$ and $g_{s y n, k}^{e}$ are the synaptic conductances in segment $k$, and $a_{k}^{i}$ and $a_{k}^{e}$ are the amplitudes of the sensory signals. The complete list of parameter values for all synapses is given in Table 6 of Appendix B. Note, that $V_{r e t, k}$ is the membrane potential of the retractor CPG neuron of the segment $k$. 

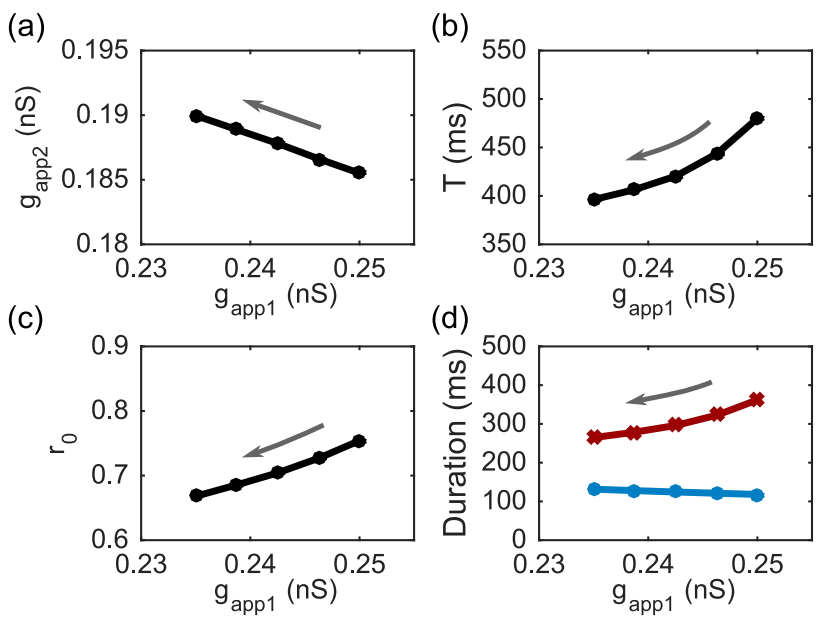

Figure 3: Effects of changing the values of the parameters $g_{a p p 1}$ and $g_{a p p 2}$ of the central drive to the CPG on its oscillatory properties. (a): linear change of $g_{a p p 1}$ and $g_{a p p 2}$ within the limits $g_{a p p 1}=0.2350 \mathrm{nS}, g_{a p p 2}=0.1900 \mathrm{nS}$ and $g_{a p p 1}=0.2500 \mathrm{nS}, g_{a p p 2}=0.1855 \mathrm{nS} ;(\mathrm{b})$ : oscillatory period $T ;(\mathrm{c})$ : duty factor $r_{0} ;(\mathrm{d})$ : length of the stance (red curve) and swing (blue line) phase. The oscillation frequency of the CPG increases in the direction shown by gray arrows.
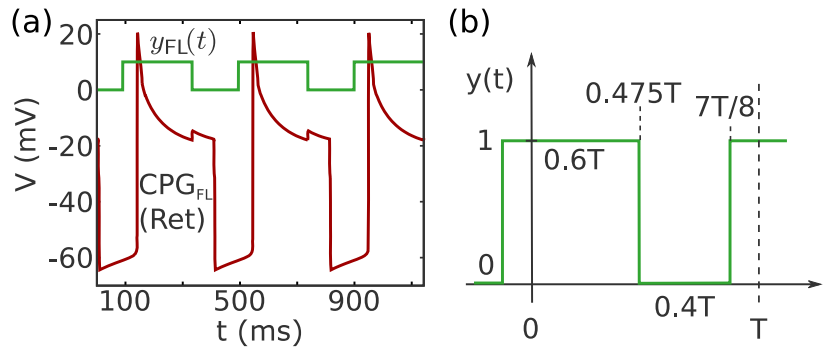

Figure 4: (a) Phase relation between the oscillatory activity of the retractor CPG neuron and the sensory signal at the same segment $y_{F L}$ (adapted from Fig. 4 in Daun-Gruhn and Tóth (2011)). (b) The rectangular signal y and its phase relation to the oscillatory activity of the protractor-retractor CPG of the same segment. The duty factor of the signal is $r_{y}=0.6$. $T$ is the period of the CPG's oscillatory activity. The stance phase starts at $t=0$. The rectangular signal has unit amplitude and a phase shift of $1 / 8$.

\section{Phase reduction}

\subsection{Phase reduction of a single CPG}

In this section, we perform phase reduction on the model of a single CPG following Proctor et al. (2010), Proctor and Holmes (2010), and Guckenheimer and Holmes (1983).

Let's consider an oscillator with an external input and a period $T$. The general equation reads

$$
\dot{\mathbf{x}}=\mathbf{f}(\mathbf{x})+\epsilon \mathbf{g}(\mathbf{x}, t), x \in \mathbb{R}^{n+1}, \quad 0<\epsilon \ll 1,
$$

where $\mathbf{g}$ is some external input. The parameter $\epsilon$ is introduced to indicate that the external input is weak.

The phase reduction of Eq. (8) is

$$
\dot{\varphi}=\omega_{0}+\left.\epsilon \sum_{j} \frac{\partial \varphi}{\partial x_{j}}\left(x_{0}(\varphi)\right) \mathrm{g}_{j}\left(\mathbf{x}_{\mathbf{0}}(\varphi), t\right)\right|_{\mathbf{x}_{\mathbf{0}} \in \Gamma_{0}},
$$


where $\varphi$ is the phase of the oscillator in a small neighborhood of $\Gamma_{0}$, an attracting hyperbolic limit cycle of Eq. (8); $\omega_{0}$ is the oscillation frequency, and $\frac{\partial \varphi}{\partial x_{j}}$ is the infinitesimal phase response curve (iPRC) (Proctor et al., 2010; Proctor and Holmes, 2010).

For the sake of simplicity, we defined the oscillation frequency as $\omega_{0}=1 / T$ and normalized the phase to take its values in the interval $[0,1]$.

We reduced every segmental CPG (i.e. each pair of mutually coupled neurons) to one phase oscillator. As discussed in the preceding section, the external input to the CPG in the inter-segmental network model was applied only in the first equation (for the membrane potential of the retractor CPG neuron) (Eq. (1)). Thus in Eq. (8), the state of the CPG was defined by the vector $\mathbf{x}=\left[V_{1}, h_{1}, V_{2}, h_{2}\right]^{\top}$. The function $\mathbf{f}(\mathbf{x})$ is the right hand side of Eq. (1) without external input. The latter is

$$
\epsilon \mathbf{g}(\mathbf{x}, t)=-\frac{1}{C_{m}}\left[\sum_{i} \mathcal{I}_{i}, 0,0,0\right]^{\top} .
$$

Then, the phase-reduced equation for a single CPG can be written as

$$
\begin{aligned}
\dot{\varphi} & =\omega_{0}+\epsilon Z_{1}(\varphi) \mathrm{g}_{1}(\mathbf{x}(\varphi), t)+\mathcal{O}\left(\epsilon^{2}\right) \\
& \approx \omega_{0}-\frac{1}{C_{m}} Z_{1}(\varphi) \sum_{i} \mathcal{I}_{i}(\mathbf{x}(\varphi), t)
\end{aligned}
$$

where $Z_{1}(\varphi)=\frac{\partial \varphi}{\partial V_{1}}$ is the first component of the iPRC (cf. Eq. (1) without external input), corresponding to the membrane potential of the retractor CPG neuron. (In Eq. (9), $\mathcal{O}\left(\epsilon^{2}\right)$ means higher order terms in $\epsilon$, which are small and can be neglected.) It should be noticed that in our case, the external input (synaptic coupling) is not small. Applicability of the phase reduction theory to this type of relaxation oscillators with fast-slow dynamics were discussed by Somers and Kopell (1993, 1995); Izhikevich (2000, 2007). They showed that if the oscillator with fast transition modulation dynamics fulfills the "compression condition" (the derivative of the slow variable is less before the jump than after it, which is the case for the CPG we consider) then the phase reduction can be applied even for strong coupling. Moreover, it was demonstrated by Somers and Kopell $(1993,1995)$ that the relaxation oscillators synchronize much more quickly than smooth (non-relaxation) oscillators.

As it was mentioned in the previous section, the CPG in the original model (Eq. (1)) is an escape type half-center oscillator. The iPRC of a CPG of this type has been analyzed in Wang and Rinzel (1992); Clewley (2011) and Zhang and Lewis (2013). An example of the iPRC produced by a single CPG is shown in Fig. 5. The details of its calculation are provided in Appendix C.

As one can see in Fig. 5, the iPRC is almost zero during the active state of the retractor CPG neuron. This corresponds to the stance phase of the step. The iPRC is non-zero only during the swing phase of the step. Thus, the phase of the CPG can not be changed by an external input during the stance (retraction) phase, and the influence of such an input is strongest close to the end of the swing (protraction) phase. The positive peak is narrow due to steepness of the synaptic activation function $s_{\infty}$ (the parameter $\gamma_{s}$ is large). These properties of iPRC for escape type half-center oscillators are explained in Zhang and Lewis (2013). In our case, the CPG is asymmetric, $g_{a p p 1} \neq g_{a p p 2}$. Thus the iPRC for retractor and protractor neurons differ $\left(Z_{1}(\varphi) \neq Z_{2}(\varphi+0.5)\right)$. Moreover, the switching occurs at $r_{0}$, and not at 0.5 as in the symmetric case considered by Zhang and Lewis (2013).

The large amplitude of the iPRC towards the end of the protraction phase implies that the CPG neuron at the end of the hyperpolarized state (the state of $\mathrm{C} 2$ neuron in Fig. 2 (b)) escapes earlier from the left knee into the active state. Due to the "compression condition" the phase reduction is still applicable in this case.

In the rest of this paper, we only consider the first component of the iPRC and the first equation of the system Eq. (1) and drop the subscripts from the notations, i.e. henceforth $Z(\varphi) \equiv Z_{1}(\varphi)$ and $V \equiv V_{1}$. 

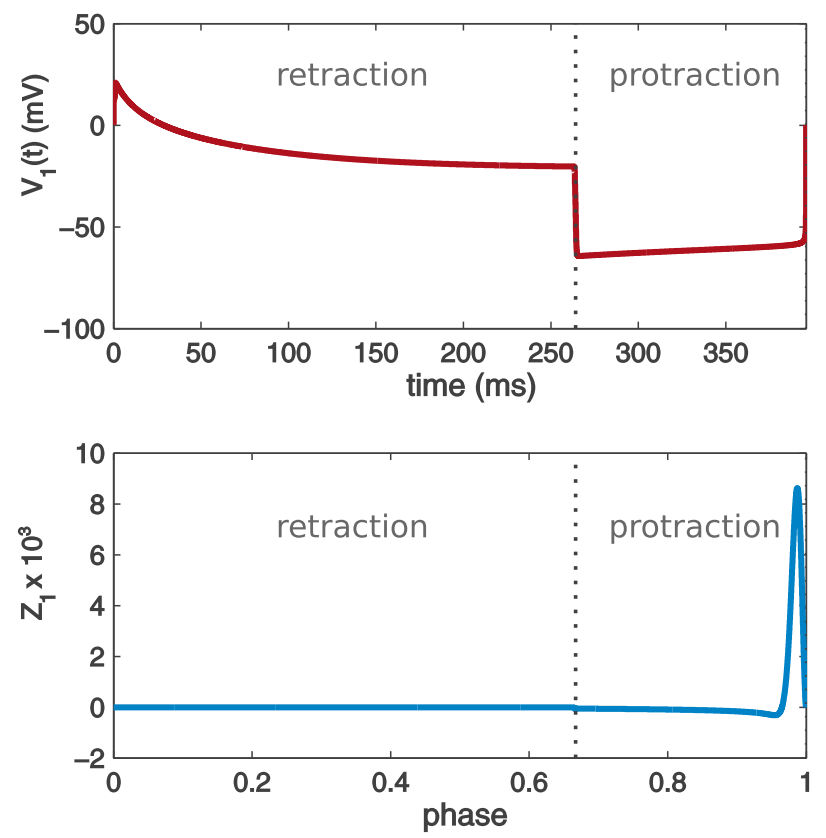

Figure 5: The infinitesimal phase response curve (iPRC) produced by a single CPG. The upper panel shows the membrane potential of the retractor neuron (red line). The bottom panel displays the corresponding component of the iPRC (blue line). The vertical dashed line indicates the instant of time at which the retraction phase switches to the protraction one.

\subsection{Application of the phase reduction method to a segmental sub-network of the inter-segmental model}

Following Eq. (9) and Eq. (7), the phase-reduced equation for a CPG of segment $k$ that receives input from the segment $l(k, l=1,2,3 ; k \neq l)$ can be written as follows

$$
\dot{\varphi}_{k}=\omega_{0}-\frac{1}{C_{m}} Z\left(\varphi_{k}\right)\left[\mathcal{I}_{k}^{i}\left(V_{k}\left(\varphi_{k}\right), V_{l}\left(\varphi_{l}\right), t\right)+\mathcal{I}_{k}^{e}\left(V_{k}\left(\varphi_{k}\right), V_{l}\left(\varphi_{l}\right), t\right)\right] .
$$

The time dependence of the input currents in Eq. (10) arises from the definition of the sensory signals in Daun-Gruhn and Tóth (2011). In the original model, the period of the sensory signals was adjusted during the simulation so that the phase of these sensory signals was shifted relative to the phase of the corresponding CPG. Therefore, in the phase oscillator model, we can replace the time variable in the functions $y_{l}^{e}(t)$ and $y_{k}^{i}(t)$ in Eq. (10) and Eq. (7) by the phase variable $\varphi_{l}$ and $\varphi_{k}$ of the corresponding CPG. This is equivalent to the assumption that the kinematics of the legs are, in essence, determined by the CPGs and thus the sensory signals arising from a given segment have the same period as the CPGs of this segment and are phase locked to them. This assumption restricts our analysis only to stable oscillations. However, it simplifies the analysis of the system having a reduced number of parameters.

Then, we can describe the sensory signals as

$$
y_{k}^{i}\left(\varphi_{k}\right)=\mathrm{y}\left(\varphi_{k}+\Delta_{k}^{i}\right), y_{l}^{e}\left(\varphi_{l}\right)=\mathrm{y}\left(\varphi_{l}+\Delta_{l}^{e}\right),
$$

where $\Delta^{i}$. and $\Delta^{e}$. are phase shifts of the sensory signals; $\mathrm{y}(\cdot)$ is a piecewise constant (rectangular) periodic function with duty factor $r_{y}=0.6$ as depicted in Fig. 4. Since the phase shifts can differ for different segments and different synaptic connections, we have six different $\Delta$ 's in total. Note, that the phase shift $\phi$ in Eq. (6) defined in Daun-Gruhn and Tóth (2011), is equivalent to $1-\Delta$; an increase in $\phi$ is the same as a decrease in $\Delta$ and vise versa. 
Finally, we obtain the phase equation for the CPG in segment $k$, connected to that in segment $l$ from Eq. (10) as

$$
\dot{\varphi_{k}}=\omega_{0}+\mathrm{h}_{k}^{i}\left(\varphi_{k}, \varphi_{l}, \Delta_{k}^{i}\right)+\mathrm{h}_{k}^{e}\left(\varphi_{k}, \varphi_{l}, \Delta_{l}^{e}\right) .
$$

where the coupling functions are

$$
\begin{aligned}
& \mathrm{h}_{k}^{i}\left(\varphi_{k}, \varphi_{l}, \Delta_{k}^{i}\right)=-g_{k}^{i} Z\left(\varphi_{k}\right) s\left(\varphi_{l}\right) \mathrm{y}\left(\varphi_{k}+\Delta_{k}^{i}\right)\left(V\left(\varphi_{k}\right)-E^{i}\right), \\
& \mathrm{h}_{k}^{e}\left(\varphi_{k}, \varphi_{l}, \Delta_{l}^{e}\right)=-g_{l}^{e} Z\left(\varphi_{k}\right) s\left(\varphi_{l}\right) \mathrm{y}\left(\varphi_{l}+\Delta_{l}^{e}\right)\left(V\left(\varphi_{k}\right)-E^{e}\right) .
\end{aligned}
$$

Here, $g_{k}^{i}=g_{s y n, k}^{i} a_{k}^{i} / C_{m}, g_{l}^{e}=g_{s y n, k}^{e} a_{l}^{e} / C_{m}$, and $s\left(\varphi_{l}\right)=s_{\infty}\left(V_{l}\left(\varphi_{l}\right)\right)$.

\subsection{Averaged phase equations}

Following Hoppensteadt and Izhikevich (1997), Proctor et al. (2010), and Proctor and Holmes (2010), we derive the averaged phase equations for two coupled CPGs. Let's introduce the phase deviation $\psi=\varphi-\omega_{0} t$ and average the phase equation (11) over the period $T$ :

$$
\begin{aligned}
\dot{\psi}_{k}= & -\frac{1}{T} \int_{0}^{T} Z\left(\psi_{k}+\omega_{0} t\right) s\left(\psi_{l}+\omega_{0} t\right) \\
& \times\left[g_{k}^{i} \mathrm{y}\left(\psi_{k}+\Delta_{k}^{i}+\omega_{0} t\right)\left(V_{k}\left(\psi_{k}+\omega_{0} t\right)-E^{i}\right)+g_{l}^{e} \mathrm{y}\left(\psi_{l}+\Delta_{l}^{e}+\omega_{0} t\right)\left(V_{k}\left(\psi_{k}+\omega_{0} t\right)-E^{e}\right)\right] \mathrm{d} t .
\end{aligned}
$$

Defining a new time variable $\tau=\psi_{k}+\omega_{0} t$, and hence $\psi_{l}+\omega_{0} t=\psi_{l}-\psi_{k}+\tau$, we have

$$
\dot{\psi}_{k}=H_{k}^{i}\left(\psi_{l}-\psi_{k}, \Delta_{k}^{i}\right)+H_{k}^{e}\left(\psi_{l}-\psi_{k}, \Delta_{l}^{e}\right),
$$

where the averaged coupling functions are

$$
H_{k}^{i}\left(\psi_{l}-\psi_{k}, \Delta_{k}^{i}\right)=-g_{k}^{i} \int_{0}^{1} Z(\tau) s\left(\psi_{l}-\psi_{k}+\tau\right) \mathrm{y}\left(\tau+\Delta_{k}^{i}\right)\left(V_{k}(\tau)-E^{i}\right) \mathrm{d} \tau
$$

and

$$
H_{k}^{e}\left(\psi_{l}-\psi_{k}, \Delta_{l}^{e}\right)=-g_{l}^{e} \int_{0}^{1} Z(\tau) s\left(\psi_{l}-\psi_{k}+\tau\right) \mathrm{y}\left(\psi_{l}-\psi_{k}+\tau+\Delta_{l}^{e}\right)\left(V_{k}(\tau)-E^{e}\right) \mathrm{d} \tau .
$$

In Fig. 6, an example of the averaged coupling functions for the excitatory (dotted red line) and inhibitory (dotted blue line) inputs, and their sum (solid black line) are shown. The figure illustrates that the excitatory input dominates, whereas the inhibitory input adds a negative shift to the total coupling function when $0<\Delta \psi \lesssim r_{0}$. However, this shift is important and ensures the stability of the zero point of the total coupling function labeled in Fig. 6 with an arrow. The inhibitory input weakly depends on the value of $\Delta^{i}$. Thus, in the subsequent analysis, we can keep $\Delta^{i}$ constant and vary $\Delta^{e}$, only.

\subsection{The complete phase oscillator model}

The complete phase oscillator model resulting from the reduction of the inter-segmental network model is shown in Fig. 7.

We derive the complete phase-reduced model for fixed values of the parameters $g_{a p p 1}$ and $g_{a p p 2}$. Since these parameters define the period and duty factor of the CPG we set their oscillatory frequencies to be equal. Then, the equations of the complete reduced model read

$$
\left\{\begin{array}{l}
\dot{\varphi}_{1}=\omega+\mathrm{h}_{1}^{i}\left(\varphi_{1}, \varphi_{3}, \Delta_{1}^{i}\right)+\mathrm{h}_{1}^{e}\left(\varphi_{1}, \varphi_{3}, \Delta_{3}^{e}\right), \\
\dot{\varphi}_{2}=\omega+\mathrm{h}_{2}^{i}\left(\varphi_{2}, \varphi_{1}, \Delta_{2}^{i}\right)+\mathrm{h}_{2}^{e}\left(\varphi_{2}, \varphi_{1}, \Delta_{1}^{e}\right), \\
\dot{\varphi}_{3}=\omega+\mathrm{h}_{3}^{i}\left(\varphi_{3}, \varphi_{2}, \Delta_{3}^{i}\right)+\mathrm{h}_{3}^{e}\left(\varphi_{3}, \varphi_{2}, \Delta_{2}^{e}\right),
\end{array}\right.
$$

where the coupling functions are defined in Eq. (12). 


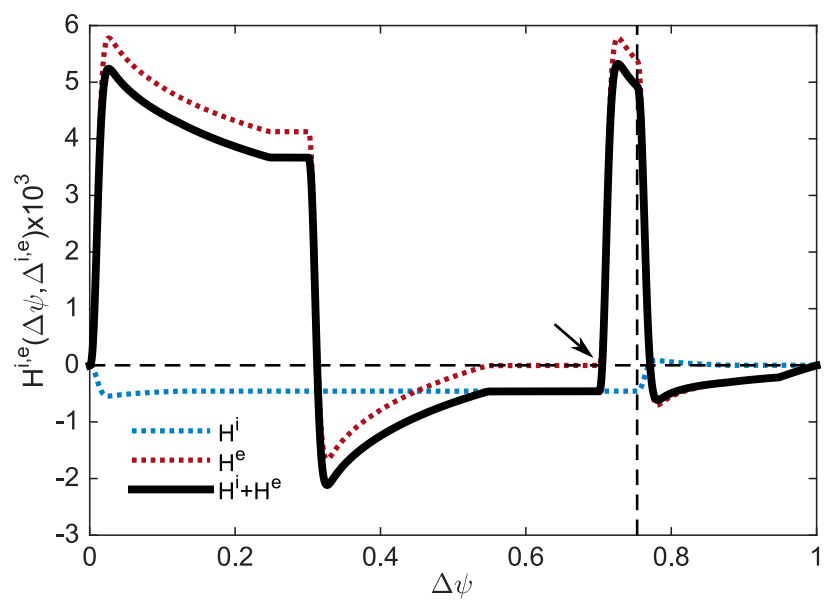

Figure 6: Examples of averaged coupling functions $H^{i}\left(\psi, \Delta^{i}\right)$ (dotted blue), $H^{e}\left(\psi, \Delta^{e}\right)$ (dotted red) and their sum (solid black). Parameters of the coupling: $\Delta^{i}=1 / 8, \Delta^{e}=0.3, g^{i}=0.327 \mathrm{~s}^{-1}, g^{e}=1.3109 \mathrm{~s}^{-1}$. Parameters of the system: $g_{a p p 1}=0.250 \mathrm{nS}, g_{a p p 2}=0.1855 \mathrm{nS}$. The vertical dashed line denotes $\psi=r_{0}$, where $r_{0}=0.7527$ is the duty factor of the CPG.

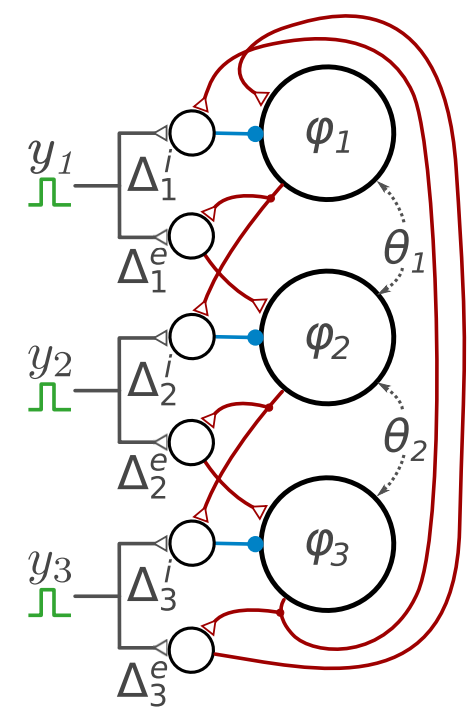

Figure 7: Phase oscillator model of the inter-segmental network model. Large black empty circles represent the protractor-retractor CPG oscillators, which are connected cyclically via sensory interneurons. The latter are depicted as small circles (cf. Fig. 1). Red lines and triangles depict excitatory connections, whereas blue lines and circles represent inhibitory connections. $y_{1}, y_{2}$, and $y_{3}$ denote sensory signals arising from the sense organs of the front, middle and the hind legs, respectively. $\Delta_{j}^{i}$ and $\Delta_{j}^{e}$ are the phase shifts between the periodic sensory signals and the oscillatory activity of the CPG of the corresponding segment $j$ in the inhibitory and excitatory synaptic connections, respectively. $\theta_{1}$ and $\theta_{2}$ are the phase differences between the first segment and the second segment and, the third segment and the second segment. For further explanations, see text.

Applying the averaging theory for phase oscillators to Eq. (16), we obtain an expression similar to Eq. (13) for all segments:

$$
\left\{\begin{array}{l}
\dot{\psi}_{1}=H_{1}^{i}\left(\psi_{3}-\psi_{1}, \Delta_{1}^{i}\right)+H_{1}^{e}\left(\psi_{3}-\psi_{1}, \Delta_{3}^{e}\right), \\
\dot{\psi}_{2}=H_{2}^{i}\left(\psi_{1}-\psi_{2}, \Delta_{2}^{i}\right)+H_{2}^{e}\left(\psi_{1}-\psi_{2}, \Delta_{1}^{e}\right), \\
\dot{\psi}_{3}=H_{3}^{i}\left(\psi_{2}-\psi_{3}, \Delta_{3}^{i}\right)+H_{3}^{e}\left(\psi_{2}-\psi_{3}, \Delta_{2}^{e}\right) .
\end{array}\right.
$$


where the averaged coupling functions $H_{k}^{i}\left(\psi_{l}-\psi_{k}, \Delta_{k}^{i}\right)$ and $H_{k}^{e}\left(\psi_{l}-\psi_{k}, \Delta_{l}^{e}\right)$ are defined in Eqs. (14) and (15).

Subtracting the 2 nd equation from the 1 st and the 3 rd one, we reduce the system to a two dimensional one

$$
\left\{\begin{aligned}
\dot{\theta}_{1}= & H_{1}^{i}\left(\theta_{2}-\theta_{1}, \Delta_{1}^{i}\right)+H_{1}^{e}\left(\theta_{2}-\theta_{1}, \Delta_{3}^{e}\right) \\
& -H_{2}^{i}\left(\theta_{1}, \Delta_{2}^{i}\right)-H_{2}^{e}\left(\theta_{1}, \Delta_{1}^{e}\right), \\
\dot{\theta}_{2}= & H_{3}^{i}\left(-\theta_{2}, \Delta_{3}^{i}\right)+H_{3}^{e}\left(-\theta_{2}, \Delta_{2}^{e}\right) \\
& -H_{2}^{i}\left(\theta_{1}, \Delta_{2}^{i}\right)-H_{2}^{e}\left(\theta_{1}, \Delta_{1}^{e}\right),
\end{aligned}\right.
$$

where $\theta_{1}=\psi_{1}-\psi_{2}$, and $\theta_{2}=\psi_{3}-\psi_{2}$, and thus $\psi_{3}-\psi_{1}=\theta_{2}-\theta_{1}$.

\subsection{Further assumptions and considerations needed for the analysis of the phase oscillator model}

The usual approach for analysis of the reduced phase oscillator model of a locomotor system (e.g. Proctor and Holmes (2010); Massarelli et al. (2016); Zhang and Lewis (2013)) is to search for phase differences that are solutions of the averaged system that represents a specific walking or swimming coordination pattern. For example, analysis of the solution of the Eq. (18) at $\left(\theta_{1}, \theta_{2}\right)=(2 / 3,1 / 3)$. In this study we aimed at understanding the mechanisms that underlie the generation of different coordination patterns in the original model. Therefore, the methods of analysis we used should provide a direct comparison of the results of the original and the reduced models.

In the previous section, we carried out phase reduction of the inter-segmental network model: every segmental CPG was reduced to a phase oscillator (Fig. 8 (a) and (c)). In the original model of Daun-Gruhn and Tóth (2011), the stance and the swing phases resulted from the active phases of the retractor and the protractor CPG neurons, respectively (see Fig. 2 and Fig. 8 (b)). Moreover, the coordination patterns produced in the simulations were determined by means of the time relation of the swing phases of different legs. However, in the phase oscillator model, the state of the CPG is defined by a single variable - the phase $\varphi$. Therefore, to compare the simulation results of the original and reduced models the stance and the swing phases in the phase oscillator model had to be defined explicitly. The stance phase was thus defined to be the interval $0 \leq \varphi<r_{0}$, and the swing phase as the interval $r_{0} \leq \varphi<1$. Here, $r_{0}$ is the duty factor of the periodic activity of a CPG with central input, only (Fig. 8 (c) and (d)). The value of $r_{0}$ is constant for fixed values of the parameters $g_{a p p 1}$ and $g_{a p p 2}$, i.e. for constant central drive. If the CPG receives additional input, the time course of the phase $\varphi$ will become nonlinear (dashed curve in Fig. 8 (d)). Nevertheless, the stance phase and the swing phase are still defined the same way as before, i.e. the end of the stance phase is where the curve hits the level $\varphi=r_{0}$. Note that this definition of the stance and swing phase is in accordance with that using the oscillatory activity of the CPG neurons in the network model, and, in turn, with the original definition given to characterize the phases of stepping in animals. In the next section, we shall further justify this definition.

Further, we performed the analysis of the reduced model in two ways. First, we consider the solution of the non-averaged system Eq. ((16)), which shows how the phases of the individual CPGs evolve for a certain set of parameter values and initial conditions. Using the definition of the stance and swing phases stated above we could replicate the walking patterns. Here, we expected that the simulation results of the non-averaged system Eq. (16) coincide with the results of the original model not only qualitatively but also quantitatively. In particular, the timing of the switching between stance and swing phases in the original and the reduced models should coincide.

To solve the non-averaged phase equation system Eq. (16) numerically, the membrane potential of the retractor neuron of the CPG $V(\varphi)$ and the $\operatorname{iPRC} Z(\varphi)$ in Eq. (12) were numerically approximated for different values of the parameters $g_{a p p 1}$ and $g_{a p p 2}$. The details of the calculation can be found in Appendix C.

Next, we investigated the averaged system Eq. (18), searching for stable solutions, i.e. coordination patterns, that are characterized by constant phase differences between the activities of the segmental CPGs. Accordingly, $\theta_{1}$ and $\theta_{2}$ should be constant in the solutions, hence the left hand side of Eq. (18) should be equal to zero. We plotted the nullclines of Eq. (18) on the $\left(\theta_{1}, \theta_{2}\right)$ surface (torus) and found their intersections, 
which are the fixed points of these equations. Note that in our case, the nullclines are closed lines due to periodicity of the variables $\theta_{1}$ and $\theta_{2}$. The eigenvalues of the Jacobian matrix of Eq. (18) evaluated at the fixed points determine the stability of the solutions at these fixed points.

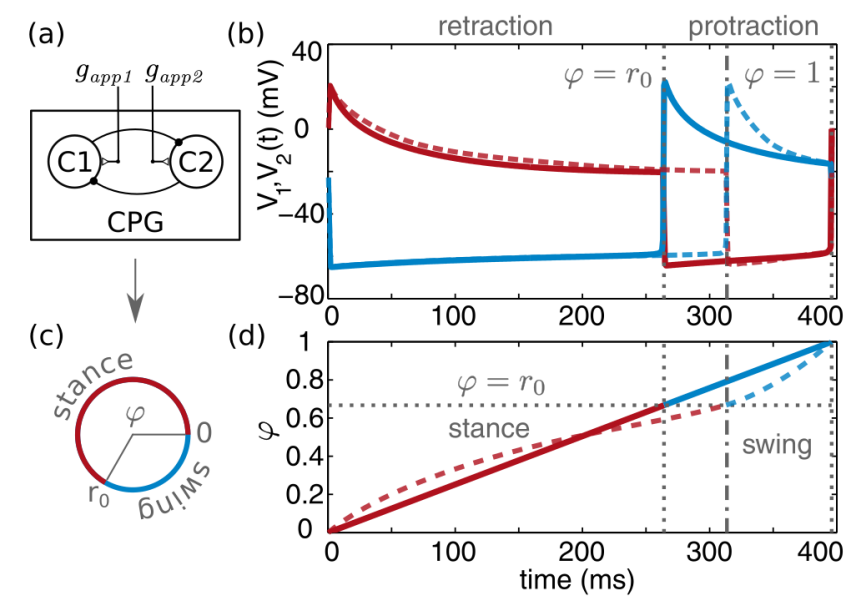

Figure 8: Scheme of phase reduction of the CPG model. (a) CPG with central input, only. (b) Membrane potential of the protractor (blue) and retractor (red) CPG neurons over one oscillatory period. An illustrative example of the time evolution of the membrane potentials in the presence of an additional input is shown with red and blue dashed lines. (c) The phase oscillator model of the CPG. (d) Stance and swing phase of the oscillation. The end of the stance phase (red) is at the point where the increasing straight line crosses the horizontal line $\varphi=r_{0}$. The rest of the period is then the swing phase. For better visibility the increasing straight line was drawn in red in the stance phase and blue in the swing phase. The dashed red-blue curve shows an illustrative example of the nonlinear time evolution of the phase $\varphi$ during an oscillatory period, if the CPG receives additional input. The end of the stance phase (the dash-dotted vertical line) is here, too, at the point where the curve intersects the horizontal line $\varphi=r_{0}$. The curve is colored the same way as the straight line.

Analysis of the fixed points of the averaged system Eq. (18) allows us to investigate the system on the reduced variables and parameters space. However, by averaging, we loose the information about the state of the individual legs, thereby the coordination pattern produced by the system. To solve this problem, we derived the regions of tripod and tetrapod coordination patterns on the $\left(\theta_{1}, \theta_{2}\right)$ plane using the definition of the stance and the swing phases (see Fig. 8). In Appendix D, we describe the details of the analysis of phase relations between $\theta_{1}=\varphi_{1}-\varphi_{2}$ and $\theta_{2}=\varphi_{3}-\varphi_{2}$, which depend on the coordination patterns.

\section{Analysis and simulations}

\subsection{Entrainment problem for two CPG oscillators}

\subsubsection{The model}

To understand the dynamics of the whole system, we started with the analysis of a model of two unidirectionally coupled segmental CPGs where one (driving) CPG oscillates with a constant frequency and entrains the second (driven) CPG. The synaptic connections between the two segments are the same as in the inter-segmental network model (see Fig. 9). According to Eq. (11) and (16) the phase equations for this model are

$$
\left\{\begin{array}{l}
\dot{\varphi}_{1}=\omega_{1} \\
\dot{\varphi}_{2}=\omega_{2}+\left[h_{2}^{i}\left(\varphi_{2}, \varphi_{1}, \Delta^{i}\right)+h_{2}^{e}\left(\varphi_{2}, \varphi_{1}, \Delta^{e}\right)\right]
\end{array}\right.
$$

where the coupling functions are defined in Eq. (12). 

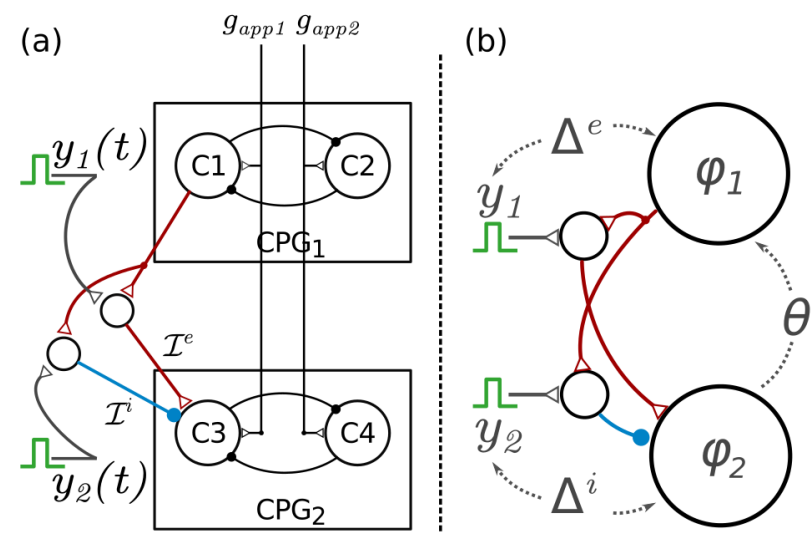

Figure 9: A model of two uni-directionally coupled segmental CPGs (a) and the corresponding phase oscillator model (b). The notations are the same as in Figs. 1 and 7.

Again, we assume that $\omega_{1}=\omega_{2}$, subtract the second equation from the first one and perform the averaging procedure. We then have

$$
\dot{\psi}_{1}-\dot{\psi}_{2}=-H_{2}^{i}\left(\psi_{1}-\psi_{2}, \Delta^{i}\right)-H_{2}^{e}\left(\psi_{1}-\psi_{2}, \Delta^{e}\right)
$$

where the functions $H_{2}^{i}$ and $H_{2}^{e}$ on the right hand side are defined in Eq. (14) and (15). As before, we introduce a new variable $\theta=\psi_{1}-\psi_{2}$ (the phase of the driving oscillator minus the phase of the driven oscillator) and obtain the equation for the phase difference

$$
\dot{\theta}=-H_{2}^{i}\left(\theta, \Delta^{i}\right)-H_{2}^{e}\left(\theta, \Delta^{e}\right)=F\left(\theta, \Delta^{i}, \Delta^{e}\right)
$$

\subsubsection{Validity of assumption}

To establish whether the assumption made in section 3.5 (see Fig. 8) on the definition of the swing and stance phases of the leg is correct, we first compared a model of two uni-directionally coupled CPGs to its reduced phase oscillator model (Eq. (19)). For this purpose, we numerically integrated the model equations of two uni-directionally coupled CPGs, and also, separately, those of the phase oscillator model (Eq. (19)). To mimic the case shown in Fig. 8 (b) and (d) we integrated both systems with similar initial conditions first without coupling, and then activated the coupling at $t=1000 \mathrm{~ms}$. Note, that the initial phases of the isolated CPGs can be uniquely defined. The parameter values in the coupling functions were the same as in the inter-segmental network model (see Appendix A and B). The phase shifts were $\Delta^{i}=1 / 8$ and $\Delta^{e}=0.3$. In the system of coupled CPGs, the parameter values of the phase shifts were chosen as $\phi^{i, e}=1-\Delta^{i, e}$. The results are shown in Fig. 10. In the two upper panels, the oscillatory activities of the two CPGs are displayed. In the bottom panel, the solution to the phase oscillator model, Eq. (19) is shown: $\varphi_{1}$ red, $\varphi_{2}$ blue oblique straight lines. Their apparent discontinuity is due to their periodicity. Using our definition of the stance and the swing phase for the oscillatory activity of the CPG neurons, we could construct a sequence of swing phases of what could be regarded as swing phases of the corresponding legs from those of the CPG oscillations. These are displayed in the middle panel of Fig. 10. One can easily see that during swing phase, the phase $\varphi$ of the corresponding phase oscillator lies between $r_{0}$ and 1 in the reduced model with activated coupling, as well as with non-activated coupling. We performed the simulation with different initial conditions and with different time points for the coupling activation and in all cases observed the same quantitative agreement between the original and reduced models. This demonstrates that the reduced model replicates the oscillatory properties of the original model and justifies the way the swing phase of a phase oscillator was defined, namely as the interval $r_{0} \leq \varphi \leq 1$. 


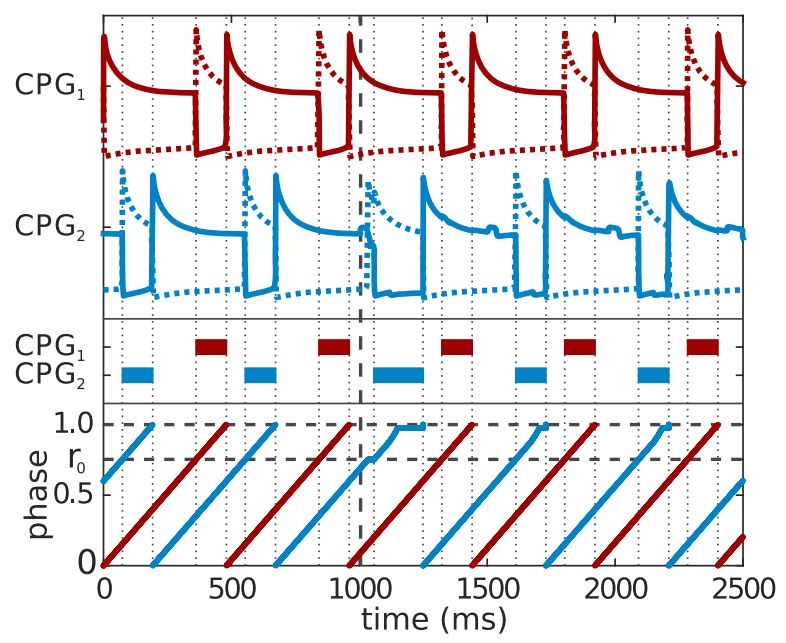

Figure 10: Comparison of a model of two uni-directionally coupled CPGs to the corresponding phase oscillator model. The coupling is activated at $t=1000 \mathrm{~ms}$ (vertical dashed line). The two upper panels show the time courses of the membrane potentials of retraction (solid) and protraction (dashed) neurons of CPG1 (red) and CPG2 (blue). In the panel below them, the red and blue bars represent the swing phases (protractions) of the corresponding legs. They were constructed from the oscillatory activity of the CPGs. In the bottom panel, the time courses of $\varphi_{1}$ and $\varphi_{2}$ in the reduced model Eq. (19) are displayed. The swing phases of the reduced model $\left(r_{0} \leq \varphi_{i} \leq 1, i=1,2\right)$ coincide with those of the original model. The discontinuity of $\varphi_{i}$ arises because of their periodicity. The boundaries of the swing phases of the CPGs are labeled by vertical dotted lines throughout all panels. The parameter values in the two uni-directionally coupled phase oscillators system Eq. (19) were $\Delta^{i}=1 / 8$ and $\Delta^{e}=0.3$; in the system of two uni-directionally coupled CPGs the phase shifts were defined as $\phi^{i, e}=1-\Delta^{i, e}$.

\subsubsection{Stability analysis}

We also performed stability analysis of the solutions to Eq. (20). In the inter-segmental network model, the phase delay in the inhibitory sensory signal $\Delta^{i}$ is constant and equal to $1 / 8$, whereas the phase shift in the excitatory sensory signal $\Delta^{e}$ is variable, and defines the coordination patterns (Daun-Gruhn and Tóth, 2011). Thus, we examined the fixed points $\theta^{*}$ of Eq. (20) using $\Delta^{e}$ as bifurcation parameter and kept $\Delta^{i}=1 / 8$ constant.

We found the fixed points $\theta^{*}$ of Eq. (20) with $g_{a p p 1}=0.2500 \mathrm{nS}, g_{a p p 2}=0.1855 \mathrm{nS}$. The bifurcation diagram is shown in Fig. 11 (a), where the stable branches are marked with black filled lines, and the unstable branches with unfilled lines. The system has four different types of stable solutions. The first type, labeled with 1 , is $\theta^{*}=0$. This solution occurs when the swing phases of both oscillators completely overlap (Fig. 11 (b)). Two other types of solutions, labeled with 2 and 3, respectively, lie on the central stable branch and can be approximated as $\theta^{*}\left(\Delta^{e}\right) \approx 1-\Delta^{e}$, if $\Delta^{e} \in\left[1-r_{0}, 1\right]$. In the interval $\Delta^{e} \in\left[1-r_{0}, r_{0}\right]$, the swing phase of the second (driven) oscillator lies between the swing phases of the first (driving) oscillator. Thus, the swing phases do not overlap (Fig. 11 (b)). This is solution type 2. If the swing phases of the oscillators partially overlap, this happens when $\Delta^{e} \in\left[r_{0}, 1\right]$, we have solution type 3 . This division of the central stable branch is formal. The last type of solutions, labeled with 4 , can be approximated as $\theta^{*} \approx r_{0}$ and is a solution with small negative latency between the swing phases of the oscillators: the swing phase of the second oscillator ends just after the swing phase of the first oscillator starts. The solutions types are summarized in Table 1.

The structure of the bifurcation diagram (Fig. 11 (a)) can be explained by means of the properties of the functions $s(\varphi), \mathrm{y}(\varphi)$, and the $\operatorname{iPRC} Z(\varphi)$. The details of derivation of approximate expressions for the stable and unstable branches are described in Appendix E. The approximate expressions imply that the stable solution $\theta^{*}$, hence, the phase difference between segments is defined primarily by the phase shift in the excitatory synaptic pathway $\Delta^{e}$ and the duty factor $r_{0}$. When $r_{0}$ decreases, the interval representing the 


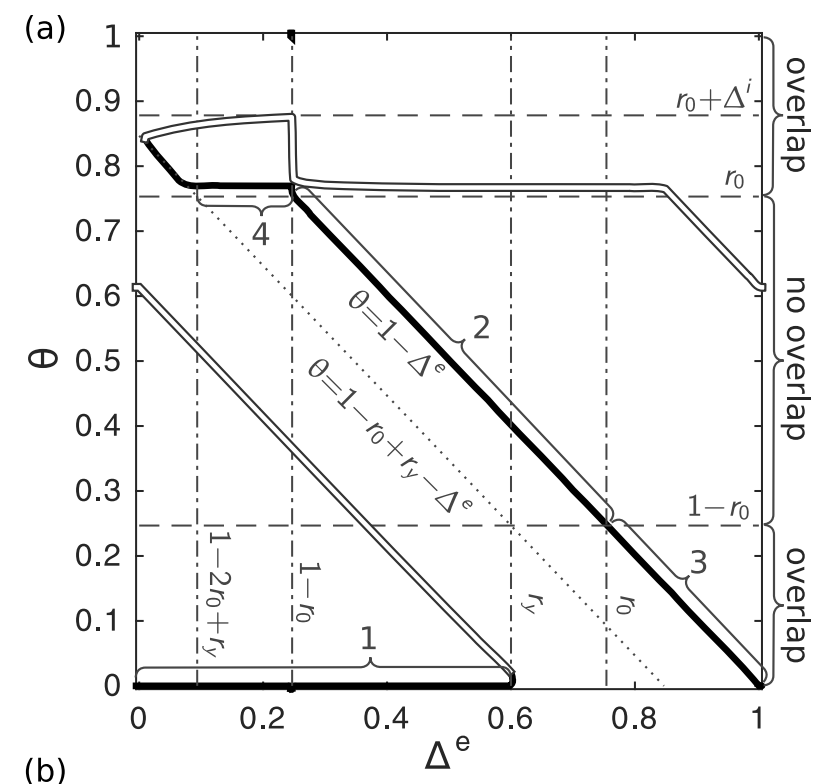

(b)

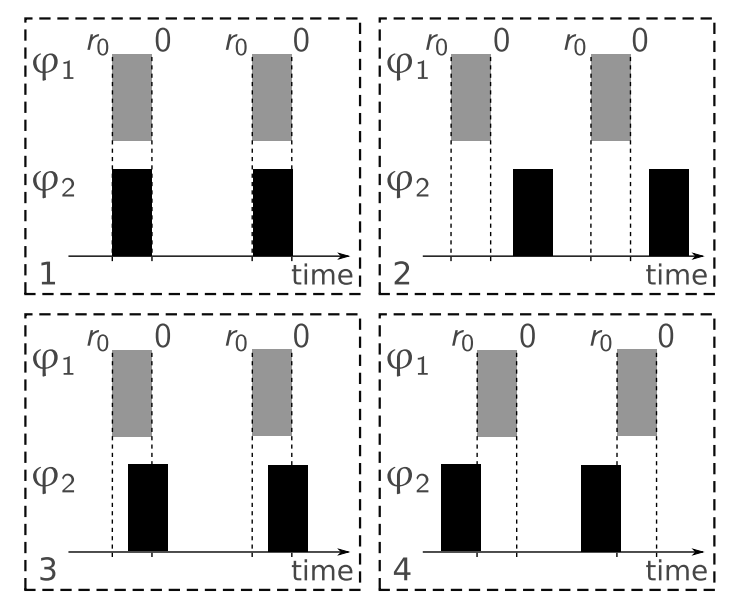

Figure 11: (a) Bifurcation diagram obtained from the averaged phase difference equation for the uni-directional coupled model (Eq. (20)): the fixed points $\theta^{*}$ of Eq. (20) versus $\Delta^{e}\left(\Delta^{i}=1 / 8\right)$. The black filled lines are the stable and the unfilled lines the unstable branches. The vertical dash-dotted lines denote $\Delta^{e}=1-2 r_{0}+r_{y}, \Delta^{e}=1-r_{0}$, $\Delta^{e}=r_{y}$, and $\Delta^{e}=r_{0}$, where $r_{0}$ and $r_{y}$ are the duty factors of the CPG and the sensory signal $y$, respectively. The horizontal lines are defined as $\theta=1-r_{0}, \theta=r_{0}$, and $\theta=r_{0}+\Delta^{i}$; the dotted oblique line as $\theta=1-r_{0}+r_{y}-\Delta^{e}$. The numbers in both panels denote the solution types. (b) Dependence of the overlap of the swing phases on the solution types. Gray and black bars symbolize the swing phases of the first and the second oscillators, respectively. Vertical dashed lines mark the start $\left(\varphi_{1}=r_{0}\right)$ and the end $\left(\varphi_{1}=0\right)$ of the swing phases of the first oscillator.

Table 1: Summary of the solution types showing the corresponding intervals of $\Delta^{e}$ and $\theta$.

\begin{tabular}{llll}
\hline & $\Delta^{e}$ & $\theta$ & $\theta\left(\Delta^{e}\right)$ \\
\hline 1 & {$\left[0, r_{y}\right]$} & 0 & 0 \\
2 & $\left(1-r_{0}, r_{0}\right]$ & {$\left[1-r_{0}, r_{0}\right)$} & $1-\Delta^{e}$ \\
3 & $\left(r_{0}, 1\right]$ & {$\left[0,1-r_{0}\right)$} & $1-\Delta^{e}$ \\
4 & $\left(1-2 r_{0}+r_{y}, 1-r_{0}\right)$ & $\approx r_{0}$ & $\approx r_{0}$ \\
\hline
\end{tabular}


solution type 4 moves down such that it remains parallel to the $\Delta^{e}$-axis, and its end points stay on the line $\theta=1-r_{0}+r_{y}-\Delta^{e}$ and $\theta=1-\Delta^{e}$ (solution line 2), respectively. However, the former line itself is shifted upwards parallel, hence the solution type 4 disappears when $r_{0}=r_{y}$. The stable branches of the solution types 1,2 , and 3 do not change with respect to $r_{0}$. Besides, the formal border between the solution types 2 and 3 , which divides the non-overlapping solution from the overlapping one, depends on $r_{0}$. Recall that the duty factor $r_{0}$ is determined by $g_{a p p 1}$ and $g_{a p p 2}$.

\subsection{The role of the phase shifts in producing stable coordination patterns in the complete system}

We have just shown that the value of $\Delta^{e}$ of the preceding segment determines the phase shift between the oscillatory activities of two segmental CPGs. The complete system (Fig. 7) consists of three inter-segmental couplings of the same kind as in the model of two uni-directionally coupled oscillators. Thus, the zeros of the $H$ functions of the system Eq. (20) correspond to constant phase differences between segments of the complete system. Using the results of the previous section, we analyzed the complete system to find different coordination patterns. First we determined the phase relations between legs in different coordination patterns using pairwise phase relation between legs to establish the values of the phase shifts $\Delta^{e}$.

Experimentally, coordination patterns can be defined and recognized by the phase shifts between the periodic movement of the legs. In the simulations, we followed this way but we used phase shifts between the oscillatory activities of the segmental CPGs instead. However, as we explained earlier in this paper, these phase shifts could directly be interpreted as ones between the movements of the different legs. We shall thus sometimes speak directly of legs and leg movements instead of (electrical) oscillatory activities of CPGs.

Let's consider examples of tetrapod and tripod coordination patterns (Fig. 12). In Fig. 12, the swing phases of FL, ML and HL are shown as black boxes. The start and end of the swing phases are drawn as dotted and dashed vertical lines, respectively. These lines are plotted relative to the swing phases of the cyclically preceding leg (e.g. FL precedes ML, and HL cyclically precedes FL). Let us consider a leg $j$ and the phase of its movement $\varphi_{j}$. Then let $\varphi_{i}$ denote the phase of the movement of the preceding leg $i$. We define the time dependent, instantaneous phase difference $\vartheta_{i j}$ as $\vartheta_{i j}=\varphi_{i}-\varphi_{j}$. This definition is analogous to that of $\theta_{k}$ and $\theta$ in the previous section. This new variable differs from $\theta_{k}$ in Eq. (18) and $\theta$ in Eq. (20), since those are time averages over one period, whereas $\vartheta_{i j}$ is instantaneous. However, the time average of $\vartheta_{i j}$ over a period of a stable coordination pattern should be equal to $\theta$.

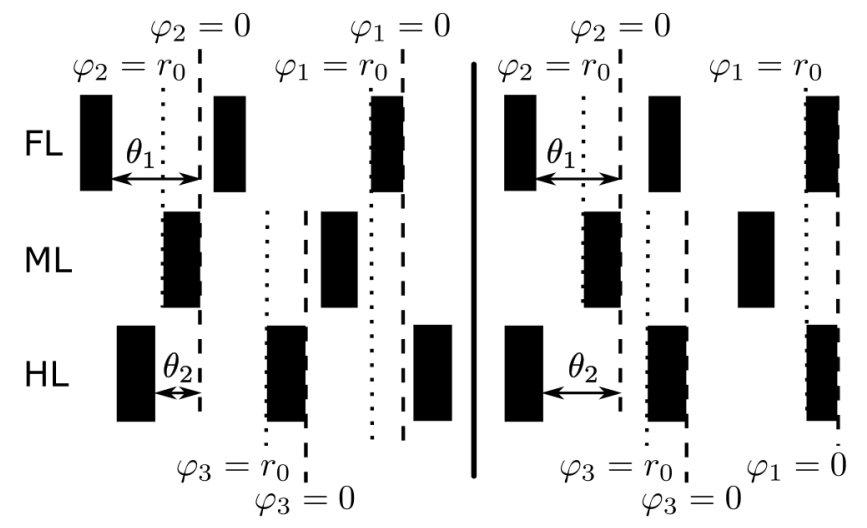

Figure 12: Examples of tetrapod (left) and tripod (right) coordination patterns and phase relations between leg movements. Black boxes represent the swing phases of the movements of FL, ML, and HL. Vertical dotted lines denote the start $\left(\varphi_{i}=r_{0}\right)$, dashed ones the end $\left(\varphi_{i}=0\right)$ of the swing phases of the leg movements. $\theta_{1}$ and $\theta_{2}$ are the phase differences defined in Eq. (20).

In order to obtain a tetrapod coordination pattern (left panel in Fig. 12), swing phases of the periodic 
movement of any leg should lie between the swing phases of the movement of the preceding leg. All phase differences should therefore obey the condition: $1-r_{0}<\vartheta_{i j}<r_{0}$. On the other hand, the value of $\vartheta_{i j}$ is determined by $\Delta_{i}^{e}$, and the solution satisfying the condition $1-r_{0}<\theta_{i}<r_{0}$, is of type 2 (see Fig. 11 and Table 1). Using the approximation for this solution type, we determined the values of the corresponding $\Delta^{e}$ 's for which a tetrapod coordination pattern is produced:

$$
r_{0}>\theta_{i}=1-\Delta_{i}^{e} \text {, thus } \Delta_{i}^{e}=1-r_{0}+\beta, i=1,2,3,
$$

where $\beta>0$ is some phase shift ensuring that this condition is satisfied. An example of the parameter values for this case is given in Table 2. It should be noted that a tetrapod coordination pattern can also be obtained if the latency between the swing phases of the leg movement is almost zero (see solution type 4 in Fig. 11 (b)). In this case, the values of $\Delta_{i}^{e}$ fall into the interval $\left[1-2 r_{0}+r_{y}, 1-r_{0}\right]$ for any pair of legs (see Table 1).

Next, we considered a tripod coordination pattern (right panel in Fig. 12). Now, the conditions for the front-to-middle and the middle-to-hind leg pairs remain the same $\left(1-r_{0}<\vartheta_{i, i+1}<r_{0}, i=1,2\right)$, whereas the swing phases of the front and hind leg movements overlap $\left(\vartheta_{31}>r_{0}\right.$ or $\left.\vartheta_{31}<1-r_{0}\right)$. Due to periodicity of the phase, these conditions are equivalent to $\left|\vartheta_{31}\right|<1-r_{0}$. Tripod coordination pattern can therefore be achieved, if we set the values of $\Delta_{1}^{e}$ and $\Delta_{2}^{e}$ as in the case of the tetrapod coordination pattern (Eq. (21)), and the value of $\Delta_{3}^{e}$ in the region of solution type 3 (see Fig. 11 and "tripod" in Table 2).

Table 2: The values of the phase shifts $\Delta_{j}^{e}$ between CPGs and modulatory signals $y$ that correspond to tetrapod (left) and tripod coordination pattern (right). The duty factor of the CPG for parameters used is $r_{0}=0.7527, \beta=0.03$. The value of $\Delta^{i}$ is $1 / 8$ for all segments.

\begin{tabular}{lll}
\hline$j$ & tetrapod & tripod \\
\hline 1 & $1-r_{0}+\beta$ & $1-r_{0}+\beta$ \\
2 & $1-r_{0}+\beta$ & $1-r_{0}+\beta$ \\
3 & $1-r_{0}+\beta$ & $r_{0}+\beta$ \\
\hline
\end{tabular}

Here, we defined tripod to be a coordination pattern with overlapping swing phases of the front and hind legs. In a "perfect" tripod coordination pattern, the swing phases of the front and hind legs are completely synchronized, i.e. $\vartheta_{31}=0$. It can be achieved by setting $\Delta_{3}^{e}$ to be in the region where solution type 1 and solution type 2 (or type 4 ) coexist. The desired phase differences are constrained by an additional condition for $\Delta_{1}^{e}$ and $\Delta_{2}^{e}$. Indeed, from the scheme of the tripod coordination pattern shown in Fig. 12 (right), we see that the front and hind leg movements are synchronous, if $\theta_{1}=\theta_{2}$. On the other hand, from the approximation of phase difference appearing in solution type 2 and the definition of $\theta_{1}$ and $\theta_{2}$, we can express them in terms of $\Delta_{1}^{e}$ and $\Delta_{2}^{e}$ :

$$
\begin{gathered}
\theta_{1}=\varphi_{1}-\varphi_{2}=\vartheta_{12}=1-\Delta_{1}^{e}, \\
\theta_{2}=\varphi_{3}-\varphi_{2}=-\vartheta_{23}=\Delta_{2}^{e}-1 .
\end{gathered}
$$

Using the periodicity of the phase, the additional condition for $\Delta_{1}^{e}$ and $\Delta_{2}^{e}$ to obtain "perfect" tripod coordination pattern is

$$
\Delta_{1}^{e}+\Delta_{2}^{e}=1
$$

This condition means that the sum of the phase shifts of the sensory signals in the front-middle and the middle-hind leg pairs add up to one oscillatory period.

In this section, we have determined the parameter values of the model to produce desired coordination patterns by calculating the phase relations between leg pairs in tetrapod and tripod coordination patterns. This results help us to understand the role of the parameters $\Delta^{e}$ in the original model. A more detailed analysis of the phase relations between all legs in different coordination patterns, and the representation of them on a two dimensional plane of phase differences is presented in Appendix D. 


\subsection{Simulation of stable stepping}

We simulated the full non-averaged system of phase oscillators (Eq. (16)) with different parameters and initial conditions. The conductances of the central input (which represents descending drive from higher brain regions) to all CPGs were equal to $g_{a p p 1}=0.2500 \mathrm{nS}, g_{a p p 2}=0.1855 \mathrm{nS}$. This produced stable oscillations with a duty factor $r_{0}=0.7530$ and period $T=477.37 \mathrm{~ms}$. The values of the other model parameters are given in Appendix A.

In the previous sections, we showed that the phase relations between segments of the model depended on the values of the phase shifts $\Delta_{j}^{e}$ between the CPG and the sensory signal $y$. We also found the values of $\Delta_{j}^{e}$ (Table 2) that correspond to different coordination patterns. Indeed, with these parameter values, the model produced stable tetrapod (Fig. 13 (a)) and tripod (Fig. 13 (b)) coordination patterns. In Fig. 13, black boxes stand for the swing phases of the legs where $\varphi_{k} \in\left[r_{0}, 1\right]$ (See Sections 3.5 and 4.1).
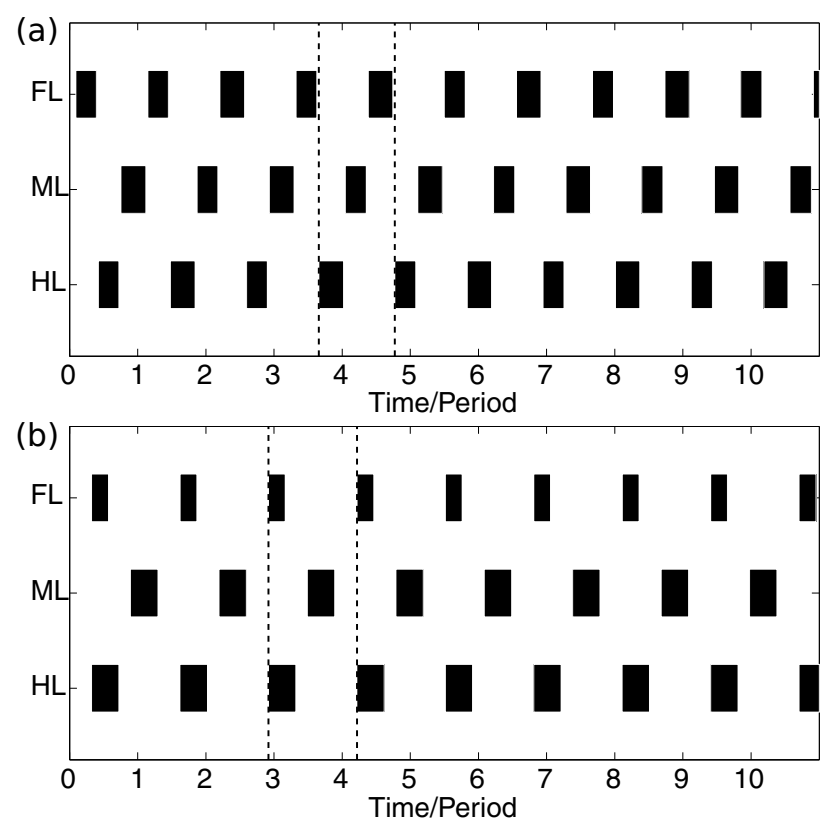

Figure 13: Tetrapod (a) and tripod (b) coordination patterns simulated by solving Eq. (16). Black bars represent the swing phases of the front (FL), middle (ML), and hind (HL) legs. The vertical dashed lines mark the start of the swing phase of the hind leg. The parameters of Eq. (16) and their values are given in Table 2.

Next, we analyzed the averaged system Eq. (18), as described in Section 3.5, with the parameter values given in Table 2. In Fig. 14, the nullclines and the fixed points of this system in the $\left(\theta_{1}, \theta_{2}\right)$ plane are shown with the regions of the coordination patterns. These regions are determined in Appendix D. A fixed point of the averaged system Eq. (18) in the $\left(\theta_{1}, \theta_{2}\right)$ plane represents a given coordination pattern. The arrows in Fig. 14 point to the stable solutions of the averaged system Eq. (18). They, in turn, correspond to the solutions of the non-averaged system Eq. (16), which are illustrated in Figs. 13. Thus a solution to the non-averaged system Eq. (16) can be associated with a fixed point in this plane. Here again, the solutions belonging to a given coordination pattern, are within the corresponding region (see Appendix D, compare with Fig. 21).

The stable focus that corresponds to the tetrapod coordination pattern is located at the intersection of two trivial nullclines of the system Eq. (18) (Fig .14 (a)). Indeed, with the parameter values given in Table 2 ("tetrapod"), the zero solution to the right hand side of the first equation of Eq. (18) is obtained, if $\theta_{2}=2 \theta_{1}$ and to that of the second equation of Eq. (18), if $\theta_{2}=-\theta_{1}$. This solution exhibits the rotational symmetry, if the phase differences between all pairs of legs are equal. The nullcline $\theta_{2}=-\theta_{1}$ also applies to the case of the tripod coordination pattern (Fig. 14 (b)). 

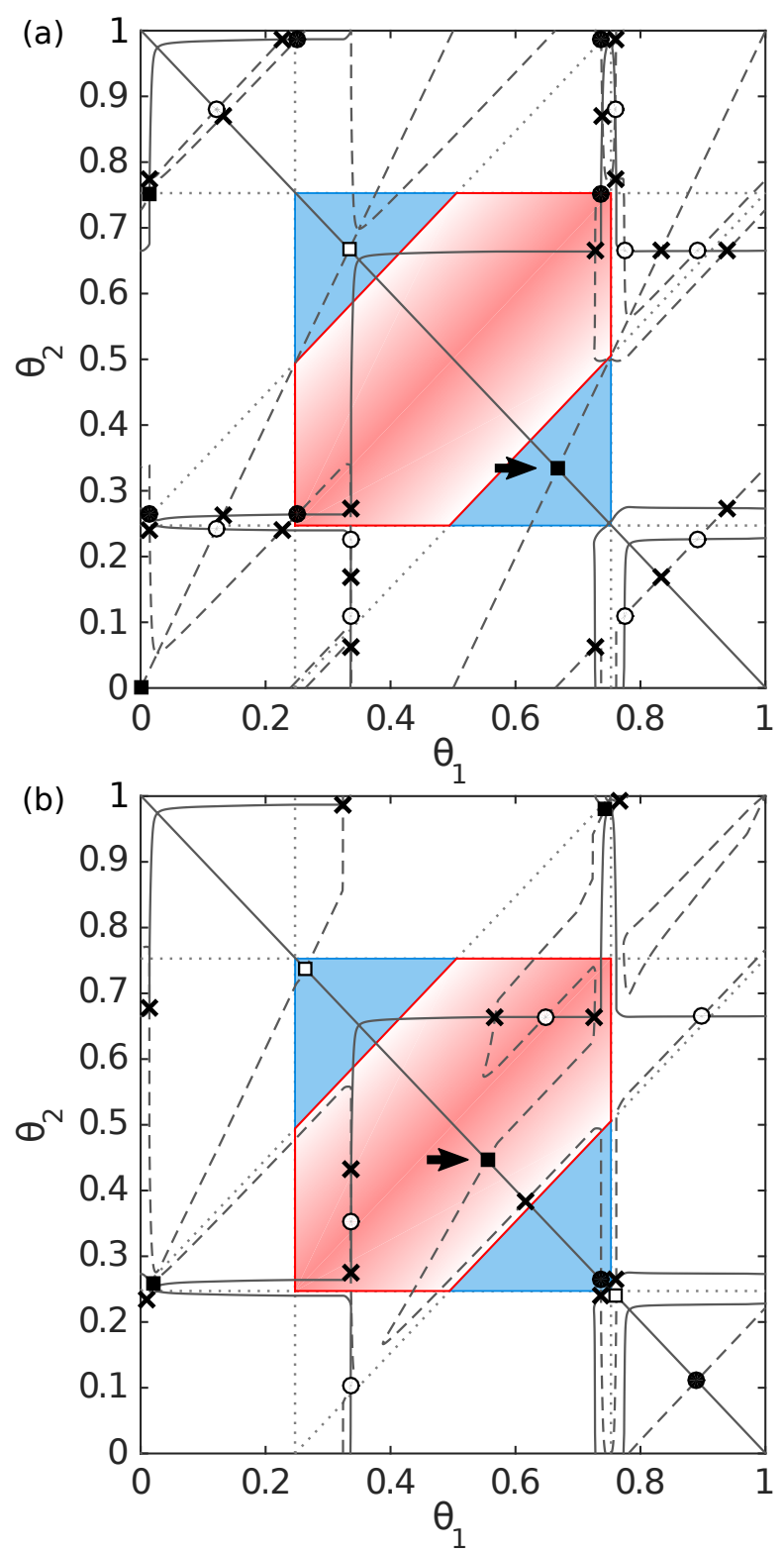

Figure 14: Nullclines and fixed points of the system Eq. (18) with the values of $\Delta_{j}^{e}$ and $\Delta_{j}^{i}$ in Table 2. The arrows point to the solutions of the averaged system Eq. (18) that correspond to the solutions of the non-averaged system Eq. (16). (a) stable tetrapod coordination pattern, (b) stable tripod coordination pattern. The blue and red areas are the regions of the tetrapod and tripod coordination patterns, respectively (see Appendix D). Dashed and solid lines are the nullclines of the first and the second equation, respectively. The different markers denote different types of fixed points: stable and unstable nodes are filled and empty circles, respectively; filled square is a stable focus; saddles are crosses.

There are other stable fixed points lying outside the regions of the tetrapod and tripod coordination patterns, i.e. inside the white area. These fixed points are solutions for which the swing phases of the neighboring legs overlap. Some of these fixed points are close to instability, like those in the upper right and the lower left corner in Fig. 14 (a) and (b). We didn't pay further attention to these solutions in the present work. However, two solutions that the model predicts need to be discussed. The first one is the 
zero solution $(0,0)$ in Fig. 14 (a), which means that all three legs are moving synchronized. The second is the solution in the lower right corner in Fig. 14 (b), which occurs when the middle and the hind legs are simultaneously in the swing phase. These solutions result in unstable postures and are not observed in the walking animal. Although the second solution can be observed for a short period of time and requires the proper coordination of the contralateral legs for stable walking (Grabowska et al., 2012). This is a limitation of the model containing only the ipsilateral legs.

We also mimicked various tripod coordination patterns using the condition Eq. (22). As expected, the simulation results revealed that for given initial conditions and for the values of $\Delta_{1}^{e}$ and $\Delta_{2}^{e}$ that satisfy Eq. (22), the swing phases of the front and the hind legs are completely synchronized (not shown). Moreover, the stable fixed point of Eq. (16) that corresponds to this "perfect" tripod lies on the central diagonal line (dashed red line in Fig. 20, Appendix D) within the region of the tripod coordination pattern. When $\Delta_{1}^{e}=\Delta_{2}^{e}=0.5$, the corresponding stable solution is in the center of the $\left(\theta_{1}, \theta_{2}\right)$ plane, at the point $(0.5,0.5)$ (not illustrated either).

\subsection{Simulation of transitions between coordination patterns}

In Daun-Gruhn and Tóth (2011), transition between coordination patterns was achieved by changing $\Delta_{3}^{e}$ by a half period without changing the oscillatory period of the CPGs. A similar transition can be performed with the reduced phase oscillator model (the non-averaged system Eq. (16)). An example of such switching from tetrapod to tripod coordination pattern and back is shown in Fig. 15. The mechanism of switching between coordination patterns can be explained by using the bifurcation diagram in Fig. 11 for the model of two coupled phase oscillators Eq. (20) (cf. Section 4.1.3). Indeed, the simulation was started with the parameter values given in Table 3 ("tetrapod") with which a stable tetrapod coordination pattern was produced. As we discussed in Sections 4.1 and 4.2, these values of the parameters $\Delta_{1}^{e}$ and $\Delta_{2}^{e}$ correspond to solution type 2 , and the value of $\Delta_{3}^{e}$ to solution type 4 (see Fig. 11). At the time point, marked by the left vertical dashed line in Fig. 15, the value of $\Delta_{3}^{e}$ was changed by a half period $(\gamma=0.5)$, whereas the values of the phase shifts $\Delta_{1}^{e}$ and $\Delta_{2}^{e}$ remained the same. The set of parameter values after this change is given in Table 3 ("tripod"). In this case, the value of $\Delta_{3}^{e}$ jumped into the interval of solution type 3 (Fig. 11), where the swing phases of the front and hind legs overlap. Thus, the reduced model switched to the stable tripod coordination pattern. In the same way, the reduced model switched back to the tetrapod solution. The switch took place at the time point marked by the right vertical dashed line in Fig. 15.

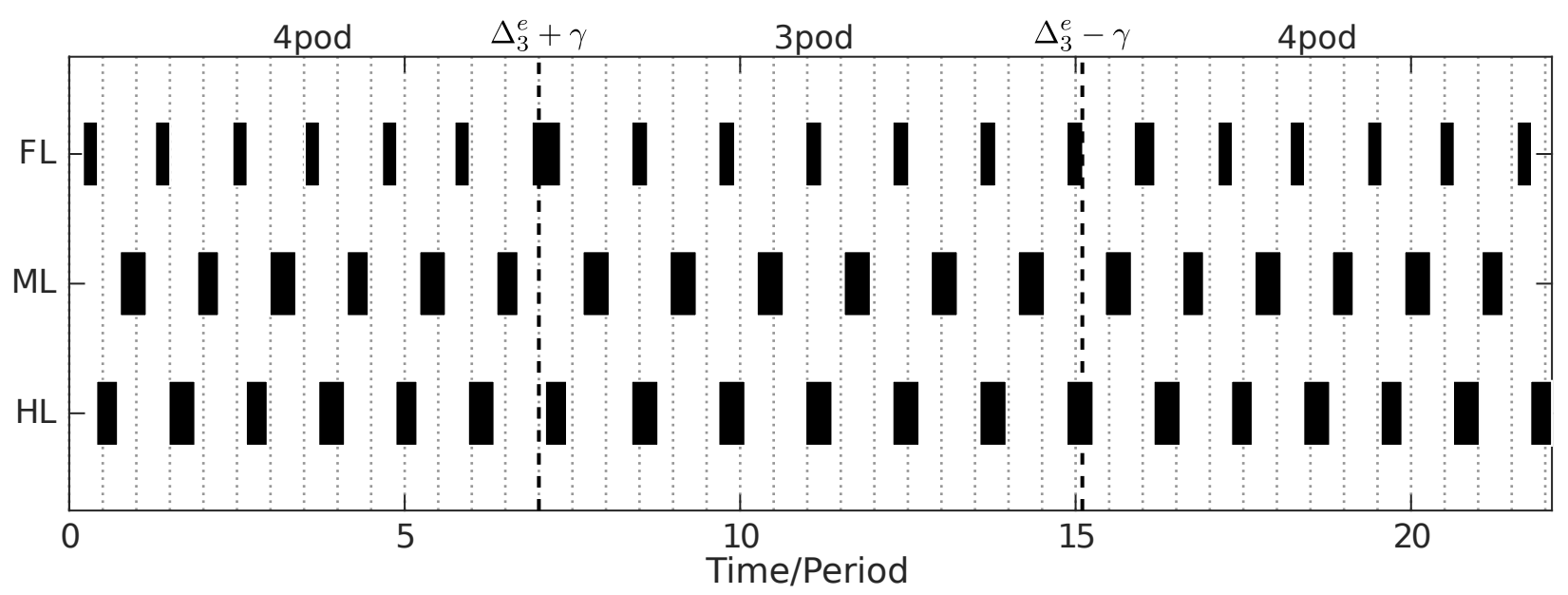

Figure 15: Illustration of the transitions from tetrapod to tripod and back by changing the phase shift $\Delta_{3}^{e}$ between the hind leg's CPG and the sensory signal $y$ by a half period $(\gamma=0.5)$. Vertical dashed lines show the time instants of the switchings. Vertical dotted gray lines indicate equidistant time points. The parameters of the non-averaged system Eq. (16) and their values are given in Table 3 
Table 3: Values of phase shifts between CPGs and modulatory signals $y$ that correspond to tetrapod (left) and tripod (right) in the simulation of switching between coordination patterns. The duty factor of the CPG for parameters used is $r_{0}=0.7527, \beta=0.03$. The value of $\Delta^{i}$ is $1 / 8$ for all segments.

\begin{tabular}{lll}
\hline$j$ & tetrapod & tripod \\
\hline 1 & $1-r_{0}+\beta$ & $1-r_{0}+\beta$ \\
2 & $1-r_{0}+\beta$ & $1-r_{0}+\beta$ \\
3 & $1-r_{0}-\beta$ & $1-r_{0}-\beta+0.5$ \\
\hline
\end{tabular}

In order to take other inter-segmental interactions in the whole system into account, we analyzed the nullclines and the fixed points of the averaged system Eq. (18) with the set of parameter values in Table 3. The resulting nullclines (not shown) were similar to those presented in Fig. 14 for stable tetrapod and tripod coordination patterns. Changing the value of $\Delta_{3}^{e}$ altered the stability and the position of the fixed points of the averaged system Eq. (18). Furthermore, at a transition between coordination patterns, the system switched from one stable solution (fixed point) in the region of one coordination pattern to a fixed point in the region of another coordination pattern.

Due to the instantaneous change of the value of $\Delta_{3}^{e}$, it is difficult to trace the time evolution of the nullclines and of the fixed points. We therefore considered another type of transition between coordination patterns in the next section. This type of transition is due to a gradual change of the period of the CPG oscillators. In this case, we could analyze the position and the stability of fixed points of the averaged system Eq. (18) in more detail.

\subsection{Transition by increasing locomotion speed}

In all examples shown in the previous sections, the central descending input to CPGs was kept constant. The isolated CPG would produce oscillatory activity with constant period and duty factor. It is, however, known (Graham, 1972; Cruse, 1990; Mendes et al., 2013) that the period and the duty factor differ in triand tetrapod coordination patterns during insect walking. In the latter case, the period and duty factor are larger than in the former. Thus, as the period of the CPG oscillation decreases, transition from tetrapod to tripod coordination pattern emerges at some point. In the model of Daun-Gruhn and Tóth (2011), this type of transition was achieved by an appropriate change of the conductances $g_{a p p 1}$ and $g_{a p p 2}$ and temporary blocking of the excitatory synapses, which lead to the phase shift between segments. We analyzed this type of transition using the reduced phase oscillator model of the inter-segmental network. We inspected the behavior of the fixed points of the averaged system Eq. (18) as the period of the CPG oscillation gradually decreased (cf. Fig. 3).

For every set of the parameter values $\left(g_{a p p 1}, g_{a p p 2}\right)$ shown in Fig. 3, we calculated the iPRC and carried out analysis of the fixed points of Eq. (18) while the values of $\Delta_{j}^{i}$ and $\Delta_{j}^{e}$ were taken from Table 2. The nullclines and fixed points are displayed in Fig. 16 for three value pairs of $\left(g_{a p p 1}\right.$ and $\left.g_{a p p 2}\right)$. As the panels of this figure demonstrate, a decreasing duty factor of the CPG and shrinking tetrapod region (upper and lower blue triangles in the two upper panels of Fig. 16) are concomitant. Moreover, if $r_{0}=2 / 3$ (bottom panel of Fig. 16), the tetrapod region altogether disappears (see also Appendix D). For larger values of $r_{0}$ (Fig. 16, uppermost panel), there exists a stable fixed point in the tetrapod region, and a stable focus in the tripod one (marked by black arrows in the panels of Fig. 16). As the duty factor decreases and the tetrapod region shrinks, the stable fixed point in that region disappears via a saddle-node bifurcation. The stable focus in the tripod region, however, survives. Thus, if the stepping starts with a low speed exerting a tetrapod coordination pattern, then, by increasing the stepping speed, a transition to tripod occurs at some speed.

In the considered case of a transition from tetrapod to tripod, the values of the phase shifts depend on the duty factor $r_{0}$, hence, on the parameters $\left(g_{a p p 1}, g_{a p p 2}\right)$. This is not a necessary condition for a transition to occur. We also analyzed the case, when the values of the phase shifts were constant (equal to the values 

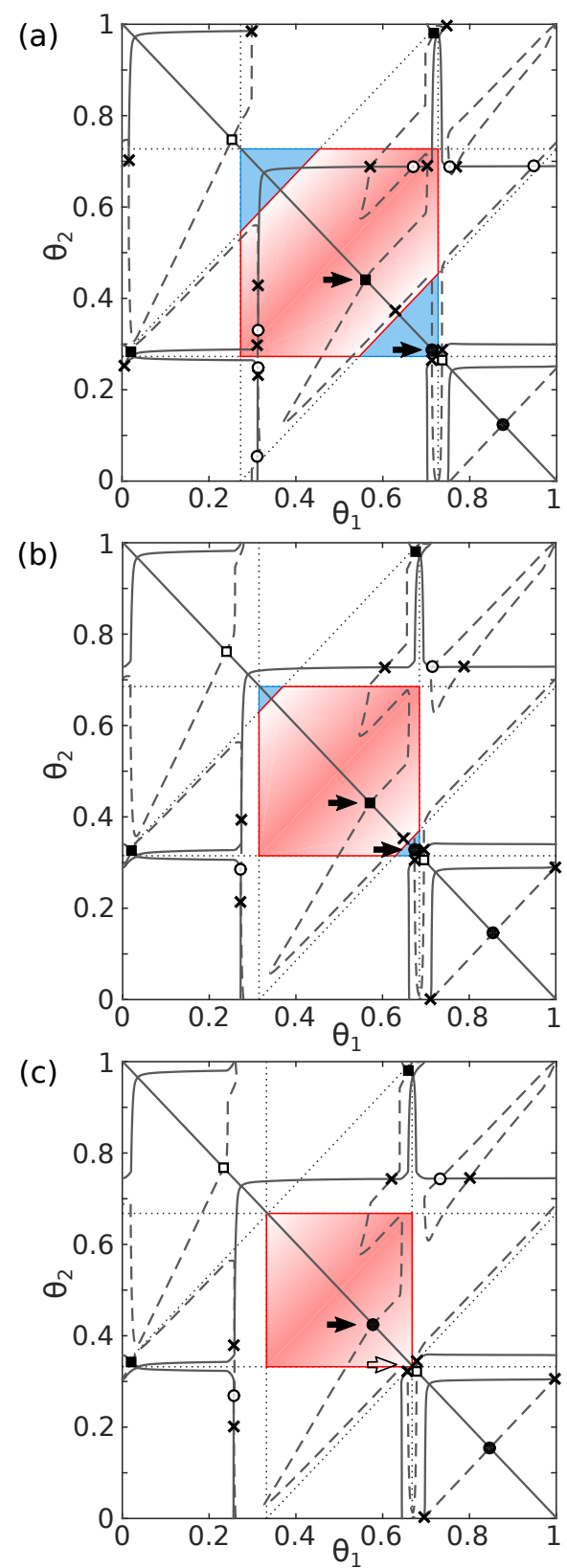

Figure 16: Nullclines and fixed points of Eq. (18) with increasing stepping speed. The values of $\left(g_{a p p 1}, g_{a p p 2}\right)$ : (a) $(0.2463,0.1866) \mathrm{nS}$, (b) $(0.2387,0.1889) \mathrm{nS}$, and (c) $(0.2350,0.1900) \mathrm{nS}$. The stable fixed point in the tetrapod region disappears (white arrow in (c)) with increasing speed, whereas the stable focus in the tripod region survives (black arrow in (c)).

of $\Delta_{j}^{i}$ and $\Delta_{j}^{e}$ in Table 2 for $\left.\left(g_{a p p 1}, g_{a p p 2}\right)=(0.2463,0.1866) \mathrm{nS}\right)$. In this case the fixed point in the tetrapod area disappears via saddle-node bifurcation at some $r_{0}>2 / 3$. Recall that the tetrapod areas disappear at $r_{0}=2 / 3$ (cf. Appendix D). 


\section{Discussion}

In this paper, we report results on the analysis of the mechanisms underlying inter-segmental coordination of stepping legs, as set out in the introduction. As a main tool, we used phase reduction models of an earlier inter-segmental network model of stick insect locomotion (Daun-Gruhn and Tóth, 2011). The original model has strong relation to experimental findings such as the pivotal role of centrally controlled CPGs and inter-segmental synaptic connections between them (Borgmann et al., 2007, 2009, 2011), modulated by peripheral sensory signals. Although itself a simplification of its biological counterpart, it could reproduce basic coordination patterns and suggested a mechanism of transition between them (Daun-Gruhn et al., 2011).

The importance of the peripheral sensory signals for coordinated walking was not only observed experimentally, but was also emphasized in other models of six-legged locomotion (Dürr et al., 2004; Schilling et al., 2013). However, due to the complexity of these systems, it is difficult to carry out a quantitative analysis of the mechanisms underlying coordinated stepping. In contrast to other phase oscillator models of six-legged locomotion (Collins and Stewart, 1993; Proctor and Holmes, 2010; Proctor et al., 2010; Aminzare et al., 2018), we dealt, after reduction, with a simplified model that still captured the effects of the sensory signals on inter-segmental coordination of the individual legs during walking.

In the process of reducing the inter-segmental network model, we first concentrated on the core of the model: the segmental protractor-retractor CPG. We thus first reduced an individual segmental CPG to a single phase oscillator. For this purpose, we calculated the iPRC for the retractor neuron of an isolated CPG. The underlying CPG model (Daun et al., 2009) is an escape-type half-center oscillator (Wang and Rinzel, 1992; Zhang and Lewis, 2013) with almost zero iPRC in the active state and with phase delay or advance in the quiescent state of the entrained CPG neuron. This indicates that the phase of the CPG oscillator, when entrained by external input to the retractor neuron, can only be changed during the protraction phase, with maximal entrainment towards its end. Insensitivity of the CPG during the stance (retraction) phase means that when a leg is on a ground, the phase of the corresponding CPG changes with a constant speed (see Fig. 10 bottom panel). This fact is also in agreement with the condition that legs on a firm ground move with a constant phase difference due to mechanical constrains.

The segmental protractor-retractor CPG in the original model is a bistable system with a fast transition between two states. As discussed by Cruse (2002), this type of half-center oscillators can effectively avoid coactivation of antagonistic muscles. To reflect this property of the $\mathrm{CPG}$, we had to explicitly separate the protraction and the retraction phases in the reduced phase oscillator model. We chose the phase of the CPG to be zero at the start of the retraction phase and normalized the oscillatory period to be one. Then the start of the protraction became the phase $r_{0}, r_{0}$ being the duty factor of the periodic oscillation of an isolated CPG.

In Section 4.1.2, we demonstrated that this definition of the swing and stance phases in the reduced phase oscillator model is appropriate and justified since it leads to a quantitative agreement between the original and the reduced model: they have the same timing of switching between stance and swing phases. On the other hand, good agreement comes from a property of a half-center oscillator with fast-slow dynamics. Indeed, the phase of an oscillator describes its state in the neighborhood of a periodic orbit (Fig 2 (b)). As it was discussed by Somers and Kopell $(1993,1995)$ and by Izhikevich (2000), a relaxation oscillator with fast threshold modulation can be successfully reduced to a phase oscillator if the "compression condition" is fulfilled. The inhibitory synaptic connections in the CPG are modeled as fast threshold modulation. Thus, our definition of the retraction and protraction phase means that $\varphi=r_{0}$ and $\varphi=0$ when the periodic orbit crosses the threshold value $V_{h s}=-43 \mathrm{mV}$ in the function $s_{\infty}(V)$ (vertical dotted line in Fig 2 (b)). Since the compression condition holds for the CPG, and the switching is fast, this definition is also valid in the presence of an external input to the CPG.

Using the definition of the stance and swing phases, we derived the regions of existence of the coordination patterns the three ipsilateral legs can produce, and illustrated them in the plane of the phase differences $\theta_{1}$ and $\theta_{2}\left(\left(\theta_{1}, \theta_{2}\right)\right.$ plane $)$. The representation of coordination patterns as regions in the $\left(\theta_{1}, \theta_{2}\right)$ plane, being derived for ipsilateral legs, does not distinguish between the wave and the tetrapod coordination patterns. However, our approach generalizes the "classical" definition of coordination patterns, which uses 
rigid phase differences between the three ipsilateral stepping legs: $(2 / 3,1 / 3)$ for tetrapod and $(1 / 2,1 / 2)$ for tripod (Graham, 1985; Wendler, 1965; Grabowska et al., 2012). Furthermore, we demonstrated that, when projecting a three dimensional trajectory of the phase oscillator model Eq. (16) that reflects a coordination pattern of stepping into the $\left(\theta_{1}, \theta_{2}\right)$ plane, the projection of this trajectory remains within the region of existence of the corresponding coordination pattern in the $\left(\theta_{1}, \theta_{2}\right)$ plane. Moreover, the regions of existence of the coordination patterns depend on the duty factor of the CPG: for $r_{0} \leq 2 / 3$, the tetrapod regions disappear but those of tripod survive. Obviously, this holds only if the swing phases of neighboring ipsilateral legs do not overlap. However, the graphical representation of the coordination patterns clearly shows why a six-legged animal walks in tripod, if its walking speed is high (the duty factor is shortened), as seen in the stick insect (Wendler, 1965) and the fly (Wosnitza et al., 2013).

The analysis of the reduced phase oscillator model of two coupled segmental CPGs yielded profound and, at the same time, somewhat surprising results. First of all, we found several types of solutions of the model equations. Furthermore, we could show that the phase difference $\theta$ between segments is determined by the duty factor $r_{0}$ (which is determined by the central drive, thus by the parameters $g_{a p p}$ ) and the phase shift $\Delta^{e}$ between the CPG activity of the preceding segment and the peripheral sensory signal arising from that segment. Thus, for some suitable sets of parameter values, an inter-segmental signal enforces early or late protraction phases in the subsequent segment that remain in a constant phase relation (see Fig. 10). This is comparable to the coordination rules 2 and 3 by Cruse (e.g. Dürr et al., 2004). As a result of this interaction between segments, a particular coordination pattern emerges in the whole system.

In Section 4.3, we demonstrated that the derived phase oscillator model Eq. (16) for the whole system was capable of reproducing the basic coordination patterns and the transition between them in a similar way as described in Daun-Gruhn and Tóth (2011). The reduced phase oscillator model of two coupled CPG oscillators (Eq. 19) provides a clear and simple explanation for the transition between coordination patterns. Such transition takes place if the system is pushed from the region of overlapping solutions into (or out of) that of not overlapping ones by changing the phase shift $\Delta^{e}$. We used the averaged equation (18) in the regions of existence of the different coordination patterns to find stable solutions of Eq. (16) associated with the different coordination patterns. The two systems of model equations are not equivalent: (18) is the simplification of the system (16). However, the stable solutions of the system (18) in the $\left(\theta_{1}, \theta_{2}\right)$ plane correspond to stable coordination patterns shown in Figs. 13 and 21. Moreover, the regions of existence of the coordination patterns are the same in both cases. The fixed points of Eq. (18) change depending on the phase shift $\Delta_{3}^{e}$. The shift of $\Delta_{3}^{e}$ affects the position and the stability of the fixed points, thus pushes the system from one region of existence of the coordination patterns into another.

In physiological terms, this means that the animal can control coordination of the legs and initiate a switch between coordination patterns using command neurons that affect the aforementioned sensory interneurons. Therefore, the phase shifts arise as a combination of the delay in the neuro-muscular feedback loop and a delay induced by descending commands. This phase-dependent control mechanism was suggested and introduced by Daun-Gruhn and Tóth (2011). The ability of the animal to "choose" the coordination pattern and switch between them was observed in experiments. For example, first-instar stick insects prefer tripod, whereas adult animals prefer tetrapod coordination patterns. The coordination pattern can also be changed depending on the walking surface (e.g. on its slope) (Grabowska et al., 2012).

We also considered various values of the parameters $g_{a p p}$. Applying them led to different oscillatory frequencies and duty factors in the activity of the CPG neurons. We changed the values of $g_{a p p}$ such that the period and the duty factor of the CPG gradually decreased. We showed that by increasing the speed of stepping, the tetrapod region shrank and, simultaneously, the stable fixed point in the tetrapod region disappeared via a saddle-node bifurcation. Thus, a transition from tetrapod to tripod could be triggered by increasing stepping speed. This finding of ours is in a very good agreement with the the experimental observations (e.g. Graham, 1972). Moreover, coexistence of tripod and tetrapod coordination patterns at the same speed ranges was also observed in stick insect (Grabowska et al., 2012) and fly walking (Wosnitza et al., 2013; Mendes et al., 2013).

A transition between coordination patterns through a saddle-node bifurcation occurring with increasing stepping speed was also observed by Aminzare et al. (2018). However, their phase oscillator model of 
cockroach locomotion also exhibits other bifurcations. This model has a different structure of the intersegmental coupling. As it was initially constructed for modeling cockroach locomotion, it does not include proprioceptive feedback. Unlike stick insects the fast-walking cockroach is expected to rely more on the patterns centrally produced by the CPGs, rather than on sensory feedback (Couzin-Fuchs et al., 2015; Ayali et al., 2015). The study of the differences in inter-leg coupling and the generation of coordination patterns of walking among various insect species therefore remains a challenging task.

In this modeling study, several assumptions were made. One of them is the assumption that the kinematics of the legs are completely driven by the segmental CPGs. Another one concerns the form of the sensory signal $y$. In general, this signal represents loading and unloading of the leg and should therefore also change its form depending on actual kinematics of the leg. Thus, excitatory as well as inhibitory influences $\left(\Delta^{e}, \Delta^{i}\right)$ would then change in accordance with it. For example, the component of the phase shift caused by the neuro-muscular mechanisms will increase with increasing the walking speed. Moreover, the sensory signal also appears to depend on the kinematics of the contralateral legs. To study these phenomena will be the topic of future research.

In summary, the main merit and novelty of the work presented here is that it has provided a comprehensive and systematic analysis of the mechanisms of inter-segmental coordination of 3 ipsilateral stepping legs.

The method of analysis used in this work, i.e. phase reduction methods together with the determination of the regions of existence for the different coordination patterns can also be extended to larger systems that include contralateral legs. Building upon the results of this study, we intend to perform comparative investigations of reduced models of different six-legged animals, such as those of stick insects, cockroaches, and flies. The results of such an analysis might reveal common mechanisms of general importance for understanding animal locomotion.

\section{Acknowledgements}

We would like to thank Philip Holmes for useful discussions in the course of the work. This research was supported by Deutsche Forschungsgemeinschaft (DFG) grants GR3690/2-1, GR3690/4-1, DA1953/5-2, and by BMBF-NSF grant 01GQ1412.

\section{A Parameters of CPG neurons}

All 6 CPG neurons have the same parameters, and their values are the same, except for those of the $g_{a p p}$ s. The CPG neurons are labeled by the numbers 1 to 6 as shown in Fig. 1. Parameters of the system Eq. (1):

Parameters of $I_{N a P}: g_{N a}=10.0 \mathrm{nS}, E_{N a}=50.0 \mathrm{mV}, V_{h m}=-37.0 \mathrm{mV}, \gamma_{m}=-0.1667 \mathrm{mV}^{-1}, V_{h h}=$ $-30.0 \mathrm{mV}, \gamma_{h}=0.1667 \mathrm{mV}^{-1}, V_{h \tau}=-30.0 \mathrm{mV}, \gamma_{\tau}=0.0833 \mathrm{mV}^{-1}, \varepsilon=0.0023 \mathrm{~ms}$.

Parameters of $I_{L}: g_{L}=2.8 \mathrm{nS}, E_{L}=-65.0 \mathrm{mV}$; and $C_{m}=0.9154 \mathrm{pF}$.

Parameters of $I_{a p p}: E_{a p p}=0.0 \mathrm{mV}$. The values of the parameters $g_{a p p 1}$ and $g_{a p p 2}$ are given in Table 4 .

Table 4: Parameters of the driving current $I_{a p p}$.

\begin{tabular}{llll}
\hline $\begin{array}{l}\text { CPG neuron } \\
\text { labels }\end{array}$ & $\begin{array}{l}\text { retractor } \\
\text { C1,C3,C5 }\end{array}$ & $\begin{array}{l}\text { protractor } \\
\text { C2,C4,C6 }\end{array}$ \\
\hline$r_{0}$ & $T_{0}, \mathrm{~ms}$ & $g_{a p p 1}, \mathrm{nS}$ & $g_{a p p 2}, \mathrm{nS}$ \\
\hline 0.6658 & 395.9 & 0.2350 & 0.1900 \\
0.7530 & 477.37 & 0.2500 & 0.1855 \\
\hline
\end{tabular}




\section{B Synaptic connections}

Here, in Tables 5 and 6 the synaptic connections in the inter-segmental network model are exhibited.

Table 5: Table of synaptic connections from CPG neuron $C_{i}$ in column $i$ to CPG neuron $C_{j}$ in row $j$. The numbers in the cells are the connection types. Their details are shown in the next table.

\begin{tabular}{l|llllll}
\hline CPG neurons & C1 & C2 & C3 & C4 & C5 & C6 \\
\hline C1 & & 1 & & & 2,3 & \\
C2 & 1 & & & & & \\
C3 & 2,3 & & & 1 & & \\
C4 & & & 1 & & & \\
C5 & & & 2,3 & & & 1 \\
C6 & & & & & 1 & \\
\hline
\end{tabular}

Table 6: Synaptic connection types. Connection type 1 is inhibitory and establishes the mutually inhibitory synaptic connections needed between the neurons of a CPG. Connection types 2 and 3 are inter-segmental connections via sensory interneurons. Connection type 2 is excitatory, whereas connection type 3 is inhibitory. $g_{s y n}$ is the conductance, $E_{s y n}$ the reversal potential of the synapse; $V_{h s}$ and $\gamma_{s}$ are the parameters of the activation of the synapse (Eq. (3)); $r_{y}$ is the phase shift between the inter-segmental and the peripheral sensory signal; and $a$ is an adjustable amplification factor.

\begin{tabular}{lllllll}
\hline Type & $\begin{array}{c}g_{\text {syn }}, \\
\mathrm{nS}\end{array}$ & $\begin{array}{c}E_{\text {syn }}, \\
\mathrm{mV}\end{array}$ & $\begin{array}{l}V_{h s}, \\
\mathrm{mV}\end{array}$ & $\begin{array}{l}\gamma_{s}, \\
\mathrm{mV}^{-1}\end{array}$ & $r_{y}$ & $a$ \\
\hline 1 & 1.0 & -80.0 & -43.0 & -10 & $\mathrm{~N} / \mathrm{A}$ & $\mathrm{N} / \mathrm{A}$ \\
2 & 0.2 & 0.0 & -43.0 & -10 & 0.6 & 6.0 \\
3 & 0.1 & -80.0 & -43.0 & -10 & 0.6 & 3.0 \\
\hline
\end{tabular}

\section{Phase response curve of a CPG}

The phase response curve (PRC) of a single CPG can be calculated by perturbing the system (Eq. (1) without external input) at different phases of the oscillatory period and finding the resulting phase shift elicited by the perturbation. In Fig. 17, an example of a PRC of a single CPG is illustrated.

If the perturbations to an oscillatory system become infinitesimally small at every phase of the oscillatory period, the resulting curve of responses to these perturbations is then called the infinitesimal phase response curve (iPRC) of the system. Its value, at every phase of the oscillatory period, can be calculated directly by solving the adjoint problem derived from the system of equations that describe the oscillator (Ermentrout, 1996; Hoppensteadt and Izhikevich, 1997; Izhikevich, 2007). This approach goes back to Malkin $(1949,1959)$. He considered a periodic oscillator $\dot{X}=f(X)$ forced by a time-dependent input $p(t)$, that obeys the following equation.

$$
\dot{X}=f(X)+\epsilon p(t)
$$

where $\epsilon \ll 1$. He formulated the following theorem:

Theorem 1 Malkin's theorem. If the unperturbed system Eq. (23) $(\epsilon=0)$ has a limit cycle with period T, then the phase of the system (23) is described by the equation

$$
\dot{\varphi}=1+\epsilon Z(\varphi) \cdot p(t),
$$



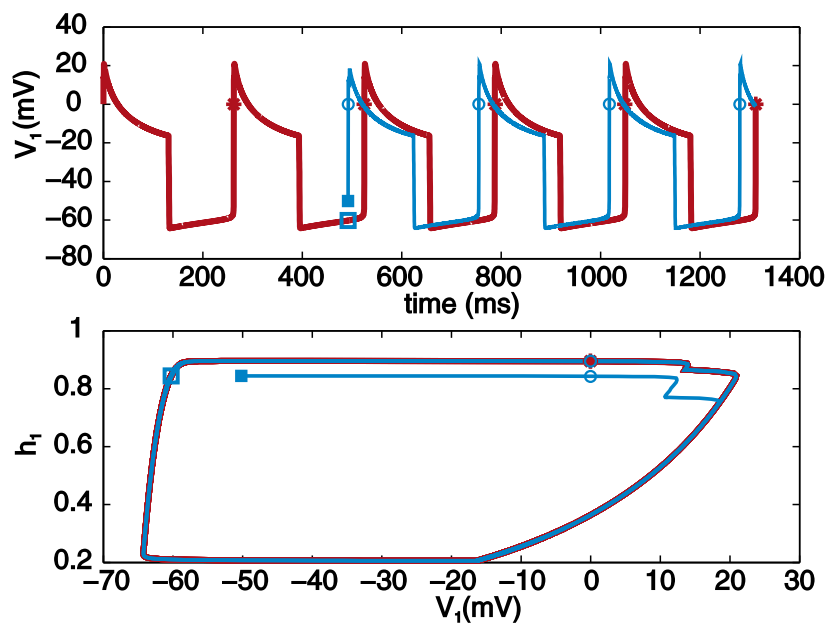

Figure 17: Calculation of PRC for a single CPG is done using the perturbation method. The top panel shows the membrane potential $\left(V_{1}\right)$ of a CPG neuron (Eq. (1)). The bottom panel displays the periodic unperturbed orbit and its perturbation in the $V-h$ plane. Red line: unperturbed (free running) system, blue line: perturbed system, empty square: before application of the perturbation, filled square: after application of the perturbation. The points with zero phase are labeled with $*$ in the unperturbed case and with a circle in the perturbed system. To get a clear effect, the strength of perturbation was chosen to be high $(10.0 \mathrm{mV})$.

where a T-periodic function $Z$ is the solution to the linear adjoint equation

$$
Z^{\prime}=-(D f)^{\top} \cdot Z
$$

subject to the normalization condition

$$
\frac{1}{T} \int_{0}^{T} Z(t) \cdot f(x(t)) \mathrm{d} t=1
$$

Here, $Z$ is the iPRC and $x(t)$ is the solution to the unperturbed system Eq. (23) $(\epsilon=0)$ on the limit cycle.

If the system $\dot{X}=f(X)$ has a periodic limit cycle with period $T$, then by rescaling the time, we can transform this equation system to a boundary value problem (BVP) of the following form

$$
\left\{\begin{array}{l}
\dot{x}-T f(x)=0, \\
x(0)-x(1)=0,
\end{array}\right.
$$

where the solution $x(t)$ is a periodic function of period 1.

To find the iPRC of a single CPG, the system (Eq. (1) without external input) and the corresponding adjoint problem (Eq. (25)) were transformed to a BVP of the form Eq. (27) and solved together with the normalization conditions (Eq. (26)) using AUTO (Doedel et al., 2007). The iPRC calculated thus is a vector of the same size as the system Eq. (1). The iPRC is almost zero in the active state of the retractor neuron of the CPG, and non-zero during its quiescent phase during which the leg is in the swing phase. This phase is thus prone to perturbations by an external input, especially just before its end.

\section{Regions of existence of coordination patterns}

We displayed several coordination patterns in Fig. 18. Depending on the sequence of stepping legs (frontmiddle-hind or hind-middle-front), there can be two different types of the tetrapod coordination pattern. 
They are denoted in Fig. 18 as tetrapod 1 and tetrapod 2. In our model, we consider ipsilateral legs (e.g. only R1, R2, and R3), only. In this case, only a possible overlap of the swing phases of the front and hind leg will distinguish the tripod coordination pattern from the tetrapod one. Thus, by determining the phase differences between the CPGs at which the swing phases of the legs overlap, we can determine the type of the coordination pattern.
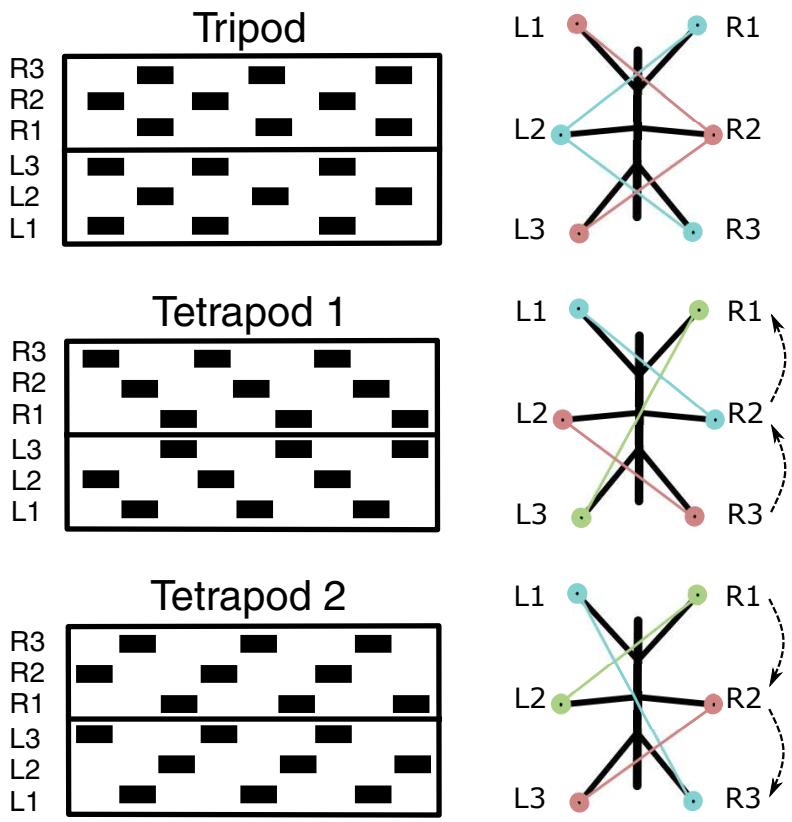

Figure 18: Tripod and tetrapod coordination patterns. R1, R2, R3: right front, middle, and hind leg; L1, L2, L3: left front, middle, and hind leg. In the panels on the left hand side, the black bars denote swing phases of the legs. The panels on the right hand side show the phase relations between the legs: lines of the same color connect the legs that have simultaneous swing phases. Tetrapod 1 and tetrapod 2 differ in the direction of swing phase sequence, as indicated by arrows.

We consider first the phase relations between two CPGs (Fig. 19). As described in Sections 3.4 and 4.1, the CPG switches from protraction to retraction at phase $\varphi=0$, and from retraction to protraction at phase $\varphi=r_{0}$. Here, $r_{0}$ is the duty factor of an isolated CPG. As pointed out earlier, the kinematics of the legs are determined by the activity of the CPG neurons. Hence, the stance and swing phase of the legs became the intervals $0 \leq \varphi<r_{0}$ and $r_{0} \leq \varphi<1$, respectively (see Fig. 8). The relative position of the swing phases of two CPGs with phases $\varphi_{j}$ and $\varphi_{k}$ with and without overlap are illustrated in Fig. 19 (a) and (b). The phase relation of two CPGs in the $\left(\varphi_{k}, \varphi_{j}\right)$ plane are displayed in Fig. 19 (c). Note that the variables $\varphi_{j}$ and $\varphi_{k}$ in this figure are periodic with a normalized period 1. From Fig. 19, we conclude that the swing phases of the CPGs won't overlap, if $1-r_{0} \leq \theta \leq r_{0}$ (the blue areas), where $\theta=\varphi_{k}-\varphi_{j}$. By contrast, if $\theta<1-r_{0}$ or $\theta>r_{0}$, which is the same as $|\theta|<1-r_{0}$, then the swing phases of two oscillators will overlap. In Fig. 19, deeper shades of red mean larger overlap. Perfect overlap occurs along the diagonal $(\theta=0)$.

The condition for the existence of the tetrapod coordination pattern is that the swing phases of any two legs should not overlap. Taking into account the definition of $\theta_{1}=\varphi_{1}-\varphi_{2}$, and $\theta_{2}=\varphi_{3}-\varphi_{2}$, we can write

$$
\begin{array}{lll}
1-r_{0} \leq \quad \theta_{1} & \leq r_{0}, \\
1-r_{0} \leq \quad \theta_{2} & \leq r_{0}, \\
1-r_{0} \leq \theta_{2}-\theta_{1} & \leq r_{0} .
\end{array}
$$

For the tripod coordination pattern, as mentioned before, the swing phases of the hind leg and the front leg overlap. Thus the last condition in Eq. (28) will be substituted by the condition for overlap:

$$
\left|\theta_{2}-\theta_{1}\right|<1-r_{0} .
$$




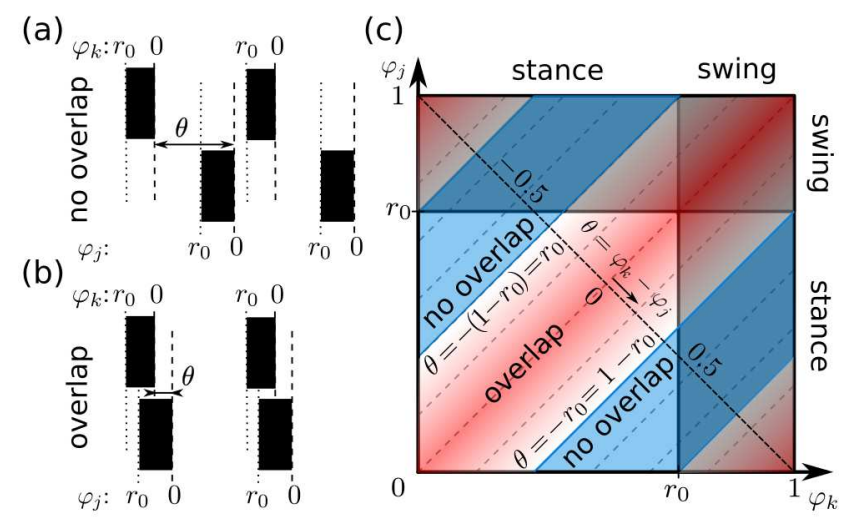

Figure 19: Phase relations between the activities of two CPGs with and without overlapping swing phases. (a) and (b): Illustration of the relative position of the swing phases (black bars). (c): The phase differences between the activities of the two CPG oscillators with phases $\varphi_{k}$ and $\varphi_{j}$ in the $\left(\varphi_{k}, \varphi_{j}\right)$ plane. The swing phases constitute the transparent gray rectangle $\left(r_{0}, 1\right] \times\left(r_{0}, 1\right]$ of the $\left(\varphi_{k}, \varphi_{j}\right)$ plane. The dashed lines parallel to the diagonal are the lines of constant phase difference: $\theta=\varphi_{k}-\varphi_{j}=$ const. In the blue regions, the swing phases of the two oscillatory activities with a constant phase difference do not overlap. The regions of overlap are drawn in varying shades of red. The deeper the shade of the red color, the greater is the overlap. Note that the region boundaries reflect the periodicity of phase variables $\varphi_{k}$ and $\varphi_{j}$.

Putting the conditions Eq. (28) and Eq. (29) together, we obtain the regions of existence of the two different coordination patterns, as illustrated in Fig. 20. The solutions to Eq. (18) in the lower blue region correspond to the coordination pattern tetrapod 1, whereas those in the upper blue triangle to the coordination pattern tetrapod 2 (see Fig. 18). The solutions in the shaded red region correspond to the tripod coordination pattern. The swing phases increasingly overlap towards the center (indicated by deeper red color in Fig. 19). Perfect overlap is achieved on the diagonal red dashed line. The white area surrounding the regions of existence of the coordination patterns represents solutions in which the swing phases of neighboring legs overlap. If the stance phase of the CPG oscillator shortens, that is the duty factor $r_{0}$ decreases, the regions of existence of tetrapod shrink. When the condition $1-r_{0}=2 r_{0}-1$ (cf. the definition of the region borders in Fig. 20) is fulfilled, i.e. $r_{0}=2 / 3$, the tetrapod solution becomes extinct.

To demonstrate the meaning of the regions of coordination patterns we projected the trajectories of the stable tetrapod and tripod coordination patterns obtained by simulation of the non-averaged phase oscillators system Eq. (16) in Section 4.3 onto the $\left(\theta_{1}, \theta_{2}\right)$ plane as shown in Fig. 21. One can see that the trajectories for a given coordination pattern do not leave the corresponding region in the plane. Note that the trajectories in the $\left(\theta_{1}, \theta_{2}\right)$ coordinate system can cross and overlap since they are projections of the three dimensional system Eq. (16) onto a two dimensional surface.

\section{E The structure of the bifurcation diagram}

The averaged coupling functions (Eq. (14) and (15)) can be approximated as sums

$$
\begin{aligned}
& H^{i}\left(\theta, \Delta^{i}\right)=g^{i} \sum_{j=1}^{N_{j}} s\left(\theta+\tau_{j}\right) \mathrm{y}\left(\Delta^{i}+\tau_{j}\right) F^{i}\left(\tau_{j}\right) \Delta \tau, \\
& H^{e}\left(\theta, \Delta^{e}\right)=g^{e} \sum_{j=1}^{N_{j}} s\left(\theta+\tau_{j}\right) \mathrm{y}\left(\theta+\Delta^{e}+\tau_{j}\right) F^{e}\left(\tau_{j}\right) \Delta \tau,
\end{aligned}
$$

where $\tau_{j} \in[0,1], \Delta \tau$ is a small increment, and the functions $F^{i}(\tau)=-Z(\tau)\left[V(\tau)-E^{i}\right]$ and $F^{e}(\tau)=$ $-Z(\tau)\left[V(\tau)-E^{e}\right]$ are depicted in Fig. 22 (a). 


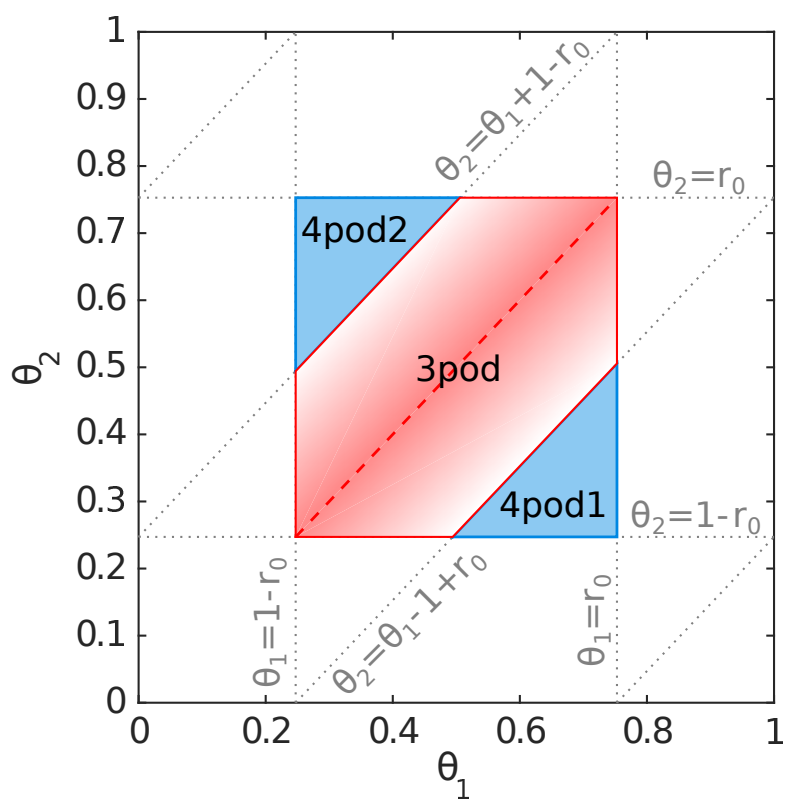

Figure 20: Illustration of the regions of existence of the coordination patterns in the $\left(\theta_{1}, \theta_{2}\right)$ plane (torus). The blue triangles are the regions of existence of tetrapod coordination patterns of type 1 and type 2 (see Fig. 18). The shaded red area is the region of existence of the tripod coordination pattern, where the swing phases of the front and hind leg overlap. The increasingly deep red color indicates increasing overlap. On the diagonal red dashed line, the overlap is perfect. The corresponding relations between $\theta_{1}$ and $\theta_{2}$ on the region boundaries (gray dotted lines) are given by Eq. (28).

We introduce a new variable $\alpha=1-\tau$ (or $\tau=-\alpha$ due to periodicity). The functions $F^{e, i}(-\alpha)$ have a large amplitude for $0<\alpha<\alpha^{*}$, where $\alpha^{*} \ll 1$ and $F^{e, i}\left(-\alpha^{*}\right)=0$. The functions $F^{e, i}$ change the sign for $\alpha^{*}<\alpha<1-r_{0}$ (see Fig. 22 (a) inset panel). For simplicity we assume that $F^{i, e}(-\alpha)=0$ for $r_{0}<\alpha \leq 1$, $s(\varphi)=1$ for $0<\varphi \leq r_{0}$, and $\mathrm{y}(\varphi)=1$ for $0<\varphi \leq r_{y}$.

For a given value of $\alpha$ the term in the sum in Eq. (31) is not zero if both $s(\theta-\alpha) \neq 0$ and $\mathrm{y}\left(\theta+\Delta^{e}-\alpha\right) \neq 0$. In the $\left(\Delta^{e}, \theta\right)$ plane (torus) these conditions are fulfilled inside the regions depicted in Fig. 22 (b) with dashed red and solid blue borders, respectively. The regions are filled with light gray color and the intersections of these regions are drawn with dark gray color. Summing up all intersection regions over the values of $\alpha$ within the range $\left[0,1-r_{0}\right]$, where $F^{e}(-\alpha) \neq 0$, gives the regions whose boundaries are shown in Fig. 22 (b) by dash-dotted black lines. Thus, inside this region $H^{e}\left(\theta, \Delta^{e}\right) \neq 0$.

The sum Eq. (31), which is the convolution of three functions $s, \mathrm{y}$ and $F^{e}$, is positive for $0<\alpha<\alpha^{*}$ due to the large positive amplitude of $F^{e}$. By contrast, within the range where the larger amplitude is not included $\left(\alpha^{*}<\alpha<1-r_{0}\right)$, the sum is negative. As $\alpha^{*} \rightarrow 0$ the regions in the $\left(\Delta^{e}, \theta\right)$ plane where $H^{e}$ is positive or negative approach the ones depicted in Fig. 22 (c) with blue and red colors, respectively. The white region is where $H^{e}\left(\theta, \Delta^{e}\right) \approx 0$.

Similarly, the function $H^{i}\left(\theta, \Delta^{i}\right)$ is a convolution of the functions $s(\theta-\alpha), \mathrm{y}\left(\Delta^{i}-\alpha\right)$, and $F^{i}(-\alpha)$ (see Eq. (30)). Since $\Delta^{i}=1 / 8$, the condition $\mathrm{y}\left(\Delta^{i}-\alpha\right) \neq 0$ reduces the range of $\alpha$ to $\left[0, \Delta^{i}\right]$. Thus, from the condition $s(\theta-\alpha) \neq 0$ we find the range $0 \leq \theta \leq r_{0}+\Delta^{i}$ where $H^{i}(\theta) \neq 0$. Again the large negative amplitude of the function $F^{i}(-\alpha)$ dominates for $0<\alpha<\alpha^{*}$. Therefor, for the limit as $\alpha^{*}=0$, the function $H^{i}$ is negative for $0<\theta \leq r_{0}$ and positive for $r_{0}<\theta \leq r_{0}+\Delta^{i}$ (see the right side of Fig. 22 (c)).

Taking into account that $\left|H^{e}\right|>\left|H^{i}\right|$ and that the functions $H^{e}$ and $H^{i}$ in Eq. (20) have negative sign, we can find the approximate expressions for the stable and unstable branches in the bifurcation diagram Fig. 11 (a) for the averaged phase difference equation of the uni-directionally coupled oscillators model (Eq. (20)):

- The central stable branch (the solution types 2 and 3) and the oblique unstable solution branch are 

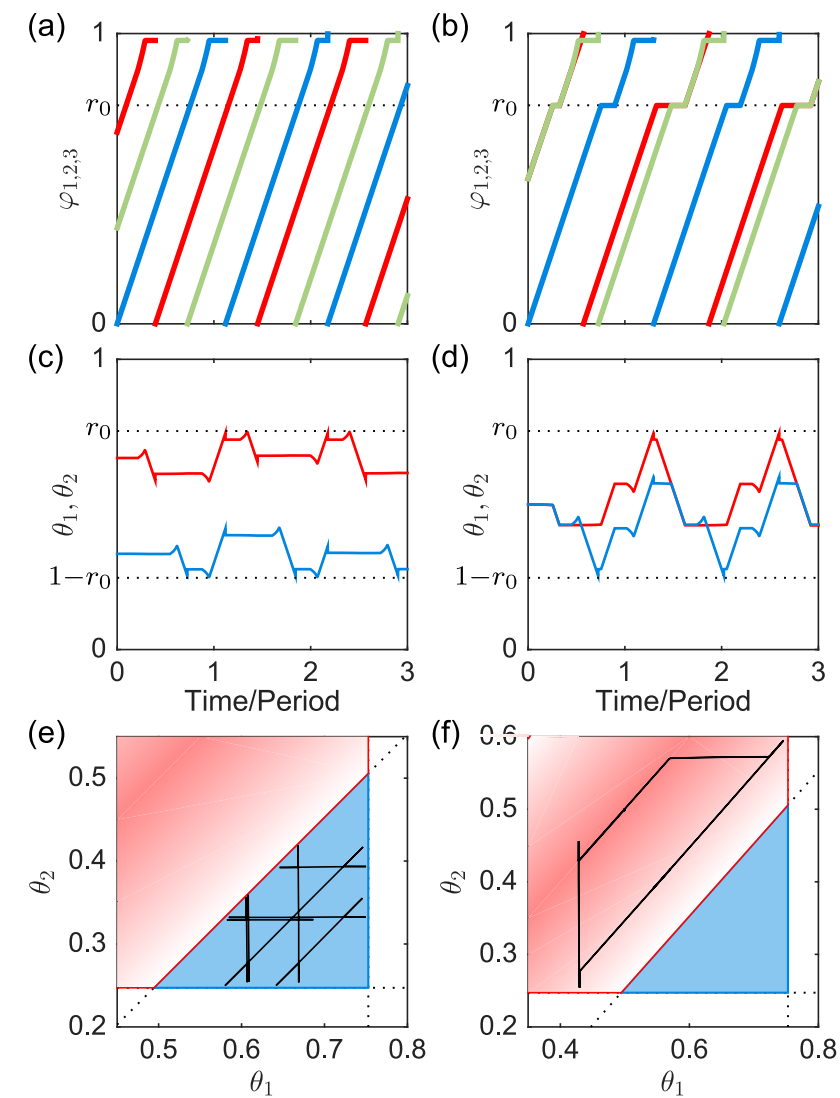

Figure 21: Projections of the trajectories of the stable tetrapod (left panels) and tripod (right panels) coordination patterns shown in Fig. 13 onto the $\left(\theta_{1}, \theta_{2}\right)$ plane. (a) and (b): Time courses of $\varphi_{1}$ (red), $\varphi_{2}$ (green), and $\varphi_{3}$ (blue) of the non-averaged system Eq. (16). (c) and (d): Time courses of the phase differences $\theta_{1}=\varphi_{1}-\varphi_{2}$ (red) and $\theta_{2}=\varphi_{3}-\varphi_{2}$ (blue) calculated by subtraction of the appropriate phase angles from the upper panels. (e) and (f): Projection of the trajectories (black) onto the $\left(\theta_{1}, \theta_{2}\right)$ plane enlarged around the corresponding region. The blue and red areas are the regions of the tetrapod and tripod coordination patterns, respectively (see Fig. 20).

the boundaries of the condition $\mathrm{y}\left(\theta+\Delta^{e}\right) \neq 0: \theta+\Delta^{e}=1$ and $\theta+\Delta^{e}=r_{y}$. Stability of the former branch is provided by the negative $H^{i}$ (cf. Fig. 6).

- The horizontal stable (the solution type 1) and unstable branch are the boundaries of the condition $s(\theta) \neq 0: \theta=0$ and $\theta=r_{0}$.

- In the upper left corner of the bifurcation diagram Fig. 11 (a) $\left(\theta \in\left[r_{0}, 1\right]\right.$ and $\left.\Delta^{e} \in\left[0,1-r_{0}\right]\right)$ the function $H^{i}$ dominates. Thus, the branch of the solution type 4 is the boundary of the condition $s(\theta) \neq 0$. The unstable branch above is bounded by the condition $s\left(\theta-\left(r_{0}+\Delta^{i}\right)\right) \neq 0: \theta=r_{0}+\Delta^{i}$.

\section{References}

Aminzare Z, Srivastava V, Holmes P (2018) Gait transitions in a phase oscillator model of an insect central pattern generator. SIAM Journal on Applied Dynamical Systems 17(1):626-671, DOI 10.1137/17M1125571

Ayali A, Couzin-Fuchs E, David I, Gal O, Holmes P, Knebel D (2015) Sensory feedback in cockroach locomotion: current knowledge and open questions. Journal of Comparative Physiology A: Neuroethology, Sensory, Neural, and Behavioral Physiology 201(9):841-850, DOI 10.1007/s00359-014-0968-1 
(a)

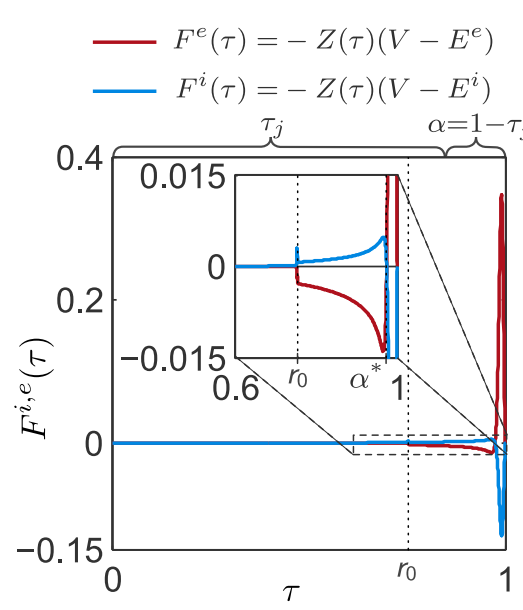

(b)

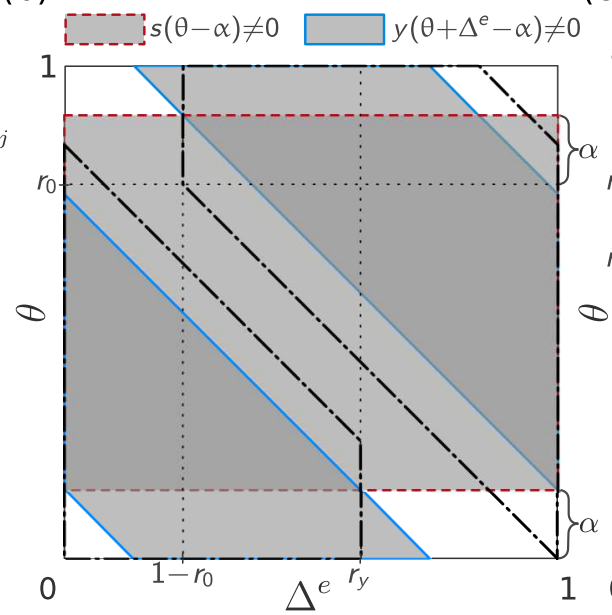

(c)

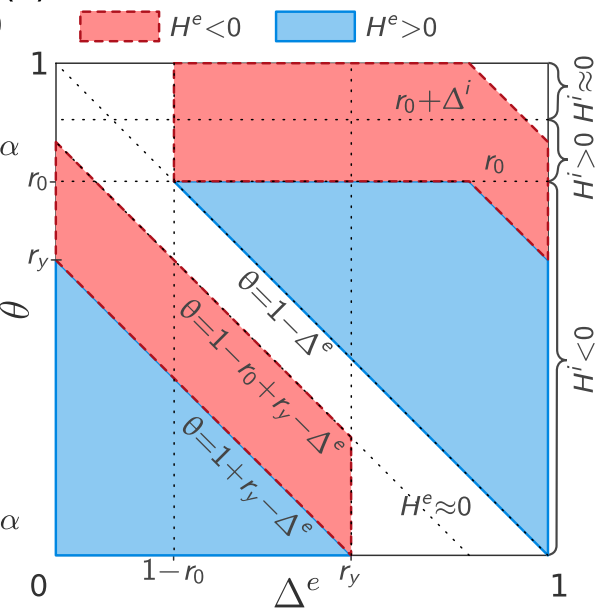

Figure 22: Illustration of the explanation of the structure of the bifurcation diagram Fig. 11. (a) Example of functions $F^{e}(\tau)$ (red line) and $F^{i}(\tau)$ (blue line). Inset panel shows the enlargement around $\left[r_{0}, 1\right]$. The vertical dotted lines denote $r_{0}$ and $\alpha^{*}\left(F^{e, i}\left(-\alpha^{*}\right)=0\right)$. (b) The regions where $s(\theta-\alpha) \neq 0$ (light gray with dashed red boundaries) and $y\left(\theta+\Delta^{e}-\alpha\right) \neq 0$ (light gray with solid blue boundaries) in the $\left(\theta, \Delta^{e}\right)$ plane for a given value of $\alpha$. The intersection of these regions are depicted with dark gray color. The regions bounded by black dash-dotted lines are the regions where $H^{e} \neq 0$, obtained by changing the value of $\alpha$ within $\left[0,1-r_{0}\right]$. (c) The regions in the $\left(\theta, \Delta^{e}\right)$ plane where the function $H^{e}>0$ (blue color with solid boundaries), $H^{e}<0$ (red color with dashed boundaries), and $H^{e} \approx 0$ (white region) in the limiting case as $\alpha^{*}=0$. The corresponding expressions for the boundaries of the regions (black dotted lines) are given. The intervals of $\theta$ when $H^{i}>0, H^{i}<0$, and $H^{i} \approx 0$ are marked on the right side.

Borgmann A, Scharstein H, Büschges A (2007) Intersegmental coordination: influence of a single walking leg on the neighboring segments in the stick insect walking system. Journal of Neurophysiology 98(3):16851696, DOI 10.1152/jn.00291.2007

Borgmann A, Hooper SL, Büschges A (2009) Sensory feedback induced by front-leg stepping entrains the activity of central pattern generators in caudal segments of the stick insect walking system. The Journal of Neuroscience 29(9):2972-2983, DOI 10.1523/JNEUROSCI.3155-08.2009

Borgmann A, Tóth TI, Gruhn M, Daun-Gruhn S, Büschges A (2011) Dominance of local sensory signals over inter-segmental effects in a motor system: experiments. Biological Cybernetics 105(5-6):399-411, DOI 10.1007/s00422-012-0473-y

Brown TG (1914) On the nature of the fundamental activity of the nervous centres; together with an analysis of the conditioning of rhythmic activity in progression, and a theory of the evolution of function in the nervous system. The Journal of Physiology 48(1):18-46, DOI 10.1113/jphysiol.1914.sp001646

Büschges A (2005) Sensory control and organization of neural networks mediating coordination of multisegmental organs for locomotion. J Neurophysiol 93(3):1127-1135, DOI 93/3/1127[pii]10.1152/jn.00615.2004

Büschges A, Gruhn M (2007) Mechanosensory Feedback in Walking: From Joint Control to Locomotor Patterns, vol 34. DOI 10.1016/S0065-2806(07)34004-6

Calabrese RL, Hill AAV, Van Hooser SD (2003) Half-center oscillators underlying rhythmic movements. In: Arbib MA (ed) Handbook of Brain Theory and Neural Networks, 2nd edn, A Bradford Book, Cambridge, pp 507-510

Clewley R (2011) Inferring and quantifying the role of an intrinsic current in a mechanism for a half-center bursting oscillation: A dominant scale and hybrid dynamical systems analysis. Journal of Biological Physics 37(3):285-306, DOI 10.1007/s10867-011-9220-1 
Cohen AH, Holmes PJ, Rand RH (1982) The nature of the coupling between segmental oscillators of the lamprey spinal generator for locomotion: A mathematical model. Journal of Mathematical Biology 13(3):345369, DOI 10.1007/BF00276069

Collins JJ, Stewart I (1993) Hexapodal gaits and coupled nonlinear oscillator models. Biological Cybernetics 68(4):287-298, DOI 10.1007/BF00201854

Couzin-Fuchs E, Kiemel T, Gal O, Ayali a, Holmes P (2015) Intersegmental coupling and recovery from perturbations in freely running cockroaches. Journal of Experimental Biology 218(2):285-297, DOI 10. $1242 /$ jeb. 112805

Cruse H (1990) What mechanisms coordinate leg movement in walking arthropods? Trends in Neurosciences 13(1):15-21, DOI 10.1016/0166-2236(90)90057-H

Cruse H (2002) The functional sense of central oscillations in walking. Biological Cybernetics 86(4):271-280, DOI 10.1007/s00422-001-0301-2

Daun S, Rubin JE, Rybak Ia (2009) Control of oscillation periods and phase durations in half-center central pattern generators: A comparative mechanistic analysis. Journal of Computational Neuroscience 27(1):336, DOI 10.1007/s10827-008-0124-4

Daun-Gruhn S (2011) A mathematical modeling study of inter-segmental coordination during stick insect walking. Journal of Computational Neuroscience 30(2):255-278, DOI 10.1007/s10827-010-0254-3

Daun-Gruhn S, Tóth TI (2011) An inter-segmental network model and its use in elucidating gait-switches in the stick insect. Journal of Computational Neuroscience 31(1):43-60, DOI 10.1007/s10827-010-0300-1

Daun-Gruhn S, Tóth TI, Borgmann A (2011) Dominance of local sensory signals over inter-segmental effects in a motor system: Modeling studies. Biological Cybernetics 105(5-6):413-426, DOI 10.1007/ s00422-012-0474-x

Delcomyn F (1980) Neural basis of rhythmic behavior in animals. Science 210(4469):492-498, DOI 10.1126/ science.7423199

Doedel EJ, Fairgrieve TF, Sandstede B, Champneys AR, Kuznetsov YA, Wang X (2007) Auto-07p: Continuation and bifurcation software for ordinary differential equations. Tech. rep., California Institute of Technology, Pasadena CA 91125, URL http://www.macs.hw.ac.uk/ \{\} gabriel/auto07/auto.html

Dürr V, Schmitz J, Cruse H (2004) Behaviour-based modelling of hexapod locomotion: Linking biology and technical application. Arthropod Structure and Development 33(3):237-250, DOI 10.1016/j.asd.2004.05. 004

Ekeberg Ö, Blümel M, Büschges A (2004) Dynamic simulation of insect walking. Arthropod Structure and Development 33(3):287-300, DOI 10.1016/j.asd.2004.05.002

Ermentrout B (1996) Type I membrances, phase resetting curves, and synchrony. Neural Computation 8(5):979-1001, DOI 10.1162/neco.1996.8.5.979

Grabowska M, Godlewska E, Schmidt J, Daun-Gruhn S (2012) Quadrupedal gaits in hexapod animals - interleg coordination in free-walking adult stick insects. Journal of Experimental Biology 215(24):4255-4266, DOI $10.1242 /$ jeb.073643

Grabowska M, Tóth TI, Smarandache-Wellmann C, Daun-Gruhn S (2015) A network model comprising 4 segmental, interconnected ganglia, and its application to simulate multi-legged locomotion in crustaceans. Journal of Computational Neuroscience 38(3):601-616, DOI 10.1007/s10827-015-0559-3 
Graham D (1972) A behavioural analysis of the temporal organisation of walking movements in the 1st instar and adult stick insect (Carausius morosus). Journal of Comparative Physiology 81(1):23-52, DOI 10.1007/BF00693548

Graham D (1985) Pattern and Control of Walking in Insects. Advances in Insect Physiology 18:31-140, DOI 10.1016/S0065-2806(08)60039-9

Guckenheimer J, Holmes P (1983) Nonlinear Oscillations, Dynamical Systems, and Bifurcations of Vector Fields. Applied Mathematical Sciences, Springer-Verlag

Holmes P, Full RJ, Koditschek D, Guckenheimer J (2006) The Dynamics of Legged Locomotion: Models, Analyses, and Challenges. SIAM Review 48(2):207-304, DOI 10.1137/S0036144504445133

Hoppensteadt FC, Izhikevich EM (1997) Weakly connected neural networks, Applied Mathematical Sciences, vol 126. Springer, DOI 10.1007/978-1-4612-1828-9

Izhikevich EM (2000) Phase Equations for Relaxation Oscillators. SIAM Journal on Applied Mathematics 60(5):1789-1804, DOI 10.1137/S0036139999351001

Izhikevich EM (2007) Dynamical Systems in Neuroscience: The Geometry of Excitability and Bursting. 1, The MIT Press

Jones SR, Mulloney B, Kaper TJ, Kopell N (2003) Coordination of cellular pattern-generating circuits that control limb movements: the sources of stable differences in intersegmental phases. The Journal of neuroscience 23(8):3457-3468

Katz PS (2016) Evolution of central pattern generators and rhythmic behaviours. Philosophical transactions of the Royal Society of London Series B, Biological sciences 371(1685):20150,057, DOI 10.1098/rstb.2015. 0057

Kukillaya RP, Holmes P (2009) A model for insect locomotion in the horizontal plane: Feedforward activation of fast muscles, stability, and robustness. Journal of Theoretical Biology 261(2):210-226, DOI 10.1016/j. jtbi.2009.07.036

Kukillaya RP, Holmes PJ (2007) A hexapedal jointed-leg model for insect locomotion in the horizontal plane. Biological Cybernetics 97(5-6):379-395, DOI 10.1007/s00422-007-0180-2

Kuramoto Y (1984) Chemical Oscillations, Waves, and Turbulence, vol 19. Springer Berlin Heidelberg, Berlin, Heidelberg, DOI 10.1007/978-3-642-69689-3

Malkin IG (1949) Methods of Poincar'e and Lyapunov in the theory of non-linear oscillations Moscow:. Moscow:Gostexizdat., [in Russian: Metodi Puankare i Liapunova v teorii nelineinix kolebanii]

Malkin IG (1959) Some problems in nonlinear oscillation theory. Moscow: Gostexizdat., [in Russian: Nekotorye zadachi teorii nelineinix kolebanii]

Massarelli N, Clapp G, Hoffman K, Kiemel T (2016) Entrainment Ranges for Chains of Forced Neural and Phase Oscillators. The Journal of Mathematical Neuroscience 6(1):6, DOI 10.1186/s13408-016-0038-9

Mendes CS, Bartos I, Akay T, Márka S, Mann RS (2013) Quantification of gait parameters in freely walking wild type and sensory deprived Drosophila melanogaster. eLife 2013(2):e00,231, DOI 10.7554/eLife.00231

Proctor J, Holmes P (2010) Reflexes and preflexes: On the role of sensory feedback on rhythmic patterns in insect locomotion. Biological Cybernetics 102(6):513-531, DOI 10.1007/s00422-010-0383-9

Proctor J, Kukillaya RP, Holmes P (2010) A phase-reduced neuro-mechanical model for insect locomotion: feed-forward stability and proprioceptive feedback. Philosophical transactions Series A, Mathematical, physical, and engineering sciences 368(1930):5087-5104, DOI 10.1098/rsta.2010.0134 
Roberts A, Roberts L B (1983) Neural origins of rhythmic movements. Cambridge University Press, Cambridge, UK

Schilling M, Hoinville T, Schmitz J, Cruse H (2013) Walknet, a bio-inspired controller for hexapod walking. Biological Cybernetics 107(4):397-419, DOI 10.1007/s00422-013-0563-5

Skinner FK, Kopell N, Mulloney B (1997) How does the crayfish swimmeret system work? Insights from nearest-neighbor coupled oscillator models. Journal of Computational Neuroscience 4(2):151-160, DOI 10.1023/A:1008891328882

Somers D, Kopell N (1993) Rapid synchronization through fast threshold modulation. Biological Cybernetics 68(5):393-407, DOI 10.1007/BF00198772

Somers D, Kopell N (1995) Waves and synchrony in networks of oscillators of relaxation and non-relaxation type. Physica D: Nonlinear Phenomena 89(1-2):169-183, DOI 10.1016/0167-2789(95)00198-0

Tóth TI, Daun-Gruhn S (2016) A three-leg model producing tetrapod and tripod coordination patterns of ipsilateral legs in the stick insect. Journal of Neurophysiology 115(2):887-906, DOI 10.1152/jn.00693.2015

Tóth TI, Grabowska M, Schmidt J, Büschges A, Daun-Gruhn S (2013a) A neuro-mechanical model explaining the physiological role of fast and slow muscle fibres at stop and start of stepping of an insect leg. PLOS ONE 8(11):e78,246, DOI 10.1371/journal.pone.0078246

Tóth TI, Schmidt J, Büschges A, Daun-Gruhn S (2013b) A neuro-mechanical model of a single leg joint highlighting the basic physiological role of fast and slow muscle fibres of an insect muscle system. PLOS ONE 8(11):e78,247, DOI 10.1371/journal.pone.0078247

Tóth TI, Grabowska M, Rosjat N, Hellekes K, Borgmann A, Daun-Gruhn S (2015) Investigating intersegmental connections between thoracic ganglia in the stick insect by means of experimental and simulated phase response curves. Biological Cybernetics (109):349-362, DOI 10.1007/s00422-015-0647-5

Von Twickel A, Hild M, Siedel T, Patel V, Pasemann F (2012) Neural control of a modular multi-legged walking machine: Simulation and hardware. Robotics and Autonomous Systems 60(2):227-241, DOI 10.1016/j.robot.2011.10.006

Wang XJ, Rinzel J (1992) Alternating and Synchronous Rhythms in Reciprocally Inhibitory Model Neurons. Neural Computation 4(1):84-97, DOI 10.1162/neco.1992.4.1.84

Wendler G (1965) The co-ordination of walking movements in arthropods. Symposium of the Society for Experimental Biology 20:229-249

Wosnitza A, Bockemuhl T, Dubbert M, Scholz H, Buschges A (2013) Inter-leg coordination in the control of walking speed in Drosophila. Journal of Experimental Biology 216(3):480-491, DOI 10.1242/jeb.078139

Zhang C, Lewis TJ (2013) Phase response properties of half-center oscillators. Journal of Computational Neuroscience 35(1):55-74, DOI 10.1007/s10827-013-0440-1

Zhang C, Lewis TJ (2016) Robust phase-waves in chains of half-center oscillators. Journal of Mathematical Biology DOI 10.1007/s00285-016-1066-5, 